\title{
TWO DIFFERENTIAL-DIFFERENCE EQUATIONS ARISING IN NUMBER THEORY
}

\author{
FERRELL S. WHEELER
} \begin{abstract}
pair of adjoint differential-difference equations:

$$
\begin{aligned}
& u p^{\prime}(u)=-a p(u)-b p(u-1), \\
& (u q(u))^{\prime}=a q(u)+b q(u+1) .
\end{aligned}
$$

ABSTRACT. We survey many old and new results on solutions of the following

We bring together scattered results usually proved only for specific $(a, b)$ pairs, while emphasizing the connections between the two equations. We also point out some of the ways these two equations are used in number theory. We give several new integral relationships between (1) and (2) and use them to prove a new application of $(2)$ in number theory, namely

$$
\sum_{\substack{1<n \leq x \\(n) \leq P_{1}(n)^{1 / u}}}\left(\log P_{1}(n)\right)^{\alpha} \sim u^{\alpha} f(u) x(\log x)^{\alpha} \quad(x \rightarrow \infty, u \geq 1, \alpha \in \mathbf{R})
$$

where $P_{1}(n)$ and $P_{2}(n)$ are the first and second largest prime divisors of $n$ and $f(u)$ satisfies $(2)$ with $(a, b)=(1-\alpha,-1)$.

\section{INTRODUCTION AND NOTATION}

In this paper we consider the two differential-difference equations

$$
\begin{aligned}
& u p^{\prime}(u)=-a p(u)-b p(u-1), \\
& (u q(u))^{\prime}=a q(u)+b q(u+1),
\end{aligned}
$$

which have arisen in various contexts in analytic number theory. Here we are using the notation of Iwaniec [I]. These two equations are said to be adjoints of each other and are frequently considered together because of the so-called adjoint relation

$$
u p(u) q(u)-b \int_{u-1}^{u} p(t) q(t+1) d t=C
$$

Received by the editors January 15,1988 .

1980 Mathematics Subject Classification (1985 Revision). Primary 11Q10, 34K99, 11N35, $11 \mathrm{~N} 25$.

Key words and phrases. Differential-difference equations, adjoint relation, sieve theory, Dickman function.

Research supported in part by a grant from the National Science Foundation. 
which holds for some constant $C$ and all sufficiently large $u$. Although a solution $p(u)$ of $(1.1)$ can have very erratic behavior, there exists a solution $q(u)$ of $(1.2)$ that is analytic in the right half-plane. Thus $q(u)$ is frequently used to study $p(u)$ using (1.3). One very typical application of (1.3) in the study of $p(u)$ is the case when $C=0$ and $q(u)$ has a root at $\alpha$ and is of one sign for $u \in(\alpha, \alpha+1)$; then letting $u=\alpha$ we see that $p(u)$ must change sign in the interval $(\alpha-1, \alpha)$.

The purpose of this paper, in general, is to give precise definitions and establish properties of two families of functions $p(u)=p(u, a, b)$ and $q(u)=$ $q(u, a, b)$ which satisfy (1.1) and (1.2), respectively. We bring together many results which have been proved only for specific coefficient pairs $(a, b)$. The emphasis will be on how these two families of functions relate to each other and how they are both used in analytic number theory.

In $\S 2$, we define $q(u)$ by means of a contour integral and briefly survey some of its properties found in [I2]. We also discuss the very important set of $q$ 's which are polynomials, namely $Q_{n}(u, b):=q(u, n+1-b, b)$, which is a polynomial in $u$ of degree $n$. Equation (1.2) is not as well known as (1.1) but, as mentioned earlier, it has been vital in studying solutions of (1.1) because of the adjoint relation (1.3). One of the most important applications of solutions of (1.2) is to provide auxiliary functions in the combinatorial sieve of dimension $\kappa>1$ due to Diamond, Halberstam, and Richert [DHR1, DHR2]. In $\S 2$ we give a detailed description of how these functions are involved in the defining relations of the fundamental parameters $\alpha_{\kappa}$ and $\beta_{\kappa}$ (the sieving limit) of the combinatorial sieve for $\kappa>1$. In $\S 10$ (Theorem 9) we show how solutions of (1.2) can arise directly in number theoretic applications. For example, if $f(u)$ is the probability (asymptotic density) that $P_{1}(n) \leq P_{2}(n)^{1 / u}$, where $P_{1}(n)$ and $P_{2}(n)$ are the first and second largest prime divisors of $n$, then $u f^{\prime}(u)=-f(u+1)$.

In $\S \S 3-6$, we consider $p(u)=p(u, a, b)$, a particular solution of $(1.1)$, for arbitrary real numbers $a$ and $b$. We bring together the work of many authors. Among other things, we discuss the behavior of $p(u)$ near its discontinuities when they exist, the Laplace transform of $p(u)$ when it exists $(\S 5)$, and the asymptotic behavior of $p(u)(\S 6)$. The function $p(u)$ arises most frequently in practice when $a+b$ is an integer, so in $\S 4$ we define $j_{\kappa}^{(n)}(u):=$ $p(u, n-\kappa, \kappa)$. We survey many of the basic properties of $j_{\kappa}(u)$ (a rescaled version of the Ankeny-Onishi-Selberg function $\sigma_{k}(u)$ ) and $j_{\kappa}^{\prime}(u)$. In $\S 7$ we also use the adjoint relation (1.3) to characterize the behavior of $j_{\kappa}^{(n)}(u)$ in relationship to its polynomial asymptote.

It has been known since the work of Dickman in 1930 and Buchstab in 1937 that retarded differential-difference equations such as (1.1) can arise in several different settings within analytic number theory. They have occurred primarily in sieve methods, in the study of incomplete sums of multiplicative functions, and in the study of integers with no large prime divisors. Probably the 
most famous example is the Dickman function $\rho(u)$ which satisfies $u \rho^{\prime}(u)=$ $-\rho(u-1)$. The easiest way to state its first appearance is that $\rho(u)$ can be thought of as the probability that the largest prime divisor of $n$ is less than $n^{1 / u}$. In $\S 8$ several explicitly stated examples of the use of $(1.1)$ in number theory are presented. In a very long paper, Levin and Fainleib [LF] study in depth the mechanism behind the occurrence of retarded differential-difference equations in certain problems of number theory. They considered a generalization of (1.1) in which more delay terms are added to the right-hand side. The corresponding adjoint was not mentioned. Since most applications seem to warrant only a single delay term, we do not go into this generalization, with the hope of obtaining more clarity.

In $\S 9$ we prove some completely new integral relationships between (1.1) and (1.2). The integral transform with kernel $t \mapsto q(t+u)$ where $0<t<+\infty$ maps solutions of (1.1) into solutions of (1.2) with a different coefficient pair $(a, b)$. A special case of this result is that the generalized Stieltjes transform of certain $p(u, a, b)$ gets mapped into solutions of (1.2). This is the mechanism behind the direct appearance of differential-difference equations of advanced type in number theoretic applications ( $\S \S 10$ and 11). Lastly, in $\S 12$ we show how the remainder term in the asymptotic expansion of $q(u, a, b)$ given by Iwaniec [12] can be expressed in terms of $j_{\kappa}^{(n)}(u)$.

Throughout this paper $\mathbf{Z}, \mathbf{Z}^{+}, \mathbf{Z}^{\leq 0}$, and $\mathbf{Z}^{\geq 0}$ denote sets containing the integers, the positive integers, the nonpositive integers, and the nonnegative integers, respectively. The letters $m$ and $n$ always denote integers and unless otherwise stated they will be positive. The letter $p$ always denotes a prime and $\pi(x)$ is the number of primes not exceeding $x$. Using standard notation (see Abramowitz and Stegun [AS] or Olver [O]), the logarithmic integral $l i(x)$, the exponential integral $E_{1}(z)$, and the complementary exponential integral $\operatorname{Ein}(z)$ are defined by the following equations:

$$
\begin{array}{rlr}
l i(x) & :=\text { p.v. } \int_{0}^{x} \frac{d t}{\log t} \quad(x>1), \\
E_{1}(z):=\int_{z}^{\infty} \frac{e^{-t}}{t} d t \quad(|\arg z|<\pi), \\
\operatorname{Ein}(z):=\int_{0}^{z} \frac{1-e^{-t}}{t} d t \quad(z \in \mathbf{C}) .
\end{array}
$$

Two frequently used relationships are

$$
\begin{array}{cc}
\operatorname{Ein}(z)=\gamma+\log z+\mathrm{E}_{1}(z) & (|\arg z|<\pi), \\
\mathrm{E}_{1}(z) \sim \frac{e^{-z}}{z}\left(1-\frac{1 !}{z}+\frac{2 !}{z^{2}} \mp \cdots\right) & (|\arg z|<\pi),
\end{array}
$$

where $\gamma=.5772 \ldots$ is Euler's constant. We remark that many of the authors 
cited have used their own notation for $\operatorname{Ein}(z)$ or variants.

2. THE FUNCTION $q(u, a, b)$

We first consider (1.2). For $u>0, a, b \in \mathbf{R}$, and $a+b \notin \mathbf{Z}^{\leq 0}$ define $q(u)=q(u, a, b)$ by

$$
q(u):=\frac{\Gamma(a+b)}{2 \pi i} \int_{\mathscr{C}} z^{-a-b} e^{u z+b \operatorname{Ein}(-z)} d z
$$

where the contour $\mathscr{C}$ has the shape

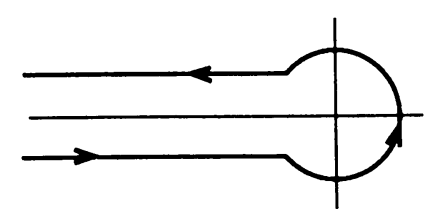

and $z^{-a-b}$ is defined using the principal branch of the logarithm. Actually, it is easy to see that $q(u, a, b)$ is analytic for $\Re u>0$ and is an entire function in each of $a$ and $b$, since equation (2.2) below shows that the singularities at $a+b \in \mathbf{Z}^{\leq 0}$ are removable. For $a, b \in \mathbf{R}$ and $u>0, q(u)$ as defined by (2.1) is real valued (this will be evident in Theorem 11) and all results will be stated for $a, b, u \in \mathbf{R}$. Many of the results could, with very little difficulty, be extended to complex variables.

Using integration by parts, it is not difficult to show that (2.1) actually satisfies (1.2) for all $u>0$ (see [I2]). We now list some of the general properties of $q(u)$ which can be found in Iwaniec [12]. For $u>0$ we have

$$
\begin{gathered}
q(u)=\frac{1}{\Gamma(1-a-b)} \int_{0}^{\infty} z^{-a-b} e^{-u z+b \operatorname{Ein}(z)} d z \quad(a+b<1), \\
q(u) \sim u^{a+b-1} \quad(u \rightarrow \infty), \\
\frac{d}{d u} q(u, a, b)=(a+b-1) q(u, a-1, b), \\
q(u)>0 \quad(a+b \leq 1 \text { or } b \leq 0), \\
\#\{\text { zeros of } q(u)\}<a+b .
\end{gathered}
$$

A special subclass of $q$ 's occurs when $a+b=n+1$ and $n \geq 0$. In this case the integrand in (2.1) has a pole of order $n+1$, instead of a branch point, at $z=0$. Thus we can collapse the straight line segments of the contour onto the negative real axis and use the residue theorem to get

$$
\begin{aligned}
q(u, n+1-b, b) & =\frac{n !}{2 \pi i} \int_{|z|=1} z^{-n-1} e^{u z+b \operatorname{Ein}(-z)} d z \\
& =n ! \operatorname{Coeff}_{z^{n}}\left(e^{u z+b \operatorname{Ein}(-z)}\right),
\end{aligned}
$$

which is a polynomial of degree $n$ in $u$. To simplify the notation somewhat, for $n \geq 0$ let

$$
Q_{n}(u, b):=q(u, n+1-b, b)
$$


which is generated by the expansion

$$
e^{u z+b \operatorname{Ein}(-z)}=\sum_{n=0}^{\infty} Q_{n}(u, b) \frac{z^{n}}{n !} .
$$

For $n \leq-1$ we will set $Q_{n}(u, b) \equiv 0$. From (2.6) we get

$$
\begin{aligned}
& Q_{0}(u, b)=1, \quad Q_{1}(u, b)=u-b, \\
& Q_{2}(u, b)=(u-b)^{2}-b / 2, \\
& Q_{3}(u, b)=(u-b)^{3}-(3 b / 2)(u-b)-b / 3,
\end{aligned}
$$

and, in general, by multiplying the Taylor series for $e^{u z}$ and $e^{v z+b \operatorname{Ein}(-z)}$, we have

$$
Q_{n}(u+v, b)=\sum_{m=0}^{n}\left(\begin{array}{c}
n \\
m
\end{array}\right) Q_{m}(v, b) u^{n-m}
$$

Also, since

$$
b \operatorname{Ein}(-z)=-b \sum_{n=1}^{\infty} \frac{z^{n}}{n n !},
$$

differentiating (2.6) with respect to $z$ with $u=0$ gives

$$
Q_{n}(0, b)=-\frac{b}{n} \sum_{m=0}^{n-1}\left(\begin{array}{c}
n \\
m
\end{array}\right) Q_{m}(0, b) \quad(n \geq 1) .
$$

Thus $Q_{n}(u, b)$ is easily computed by combining this recurrence with (2.7) (set $v=0)$.

A somewhat more interesting recurrence relation for $Q_{n}(u, b)$ is

$$
Q_{n}(u, b)=n \int_{0}^{u} Q_{n-1}(t, b) d t-b \int_{0}^{1} Q_{n-1}(t, b) d t \quad(n \geq 1) .
$$

To see this, note that (2.4) with $a=n+1-b$ implies

$$
Q_{n}(u, b)=n \int_{0}^{u} Q_{n-1}(t, b) d t+Q_{n}(0, b) .
$$

Combining this with (1.2) we have

$$
(n-b) Q_{n}(0, b)=-b Q_{n}(1, b)=-b n \int_{0}^{1} Q_{n-1}(t, b) d t-b Q_{n}(0, b) .
$$

The result follows after solving for $Q_{n}(0, b)$.

Apart from the case $a+b=n+1, q(u)$ has a simple structure in another case, namely when $b=0$. We have $q(u, a, 0)=u^{a-1}$, which follows easily either from the differential equation (1.2) or from the definition (2.1) using Hankel's contour integral for $1 / \Gamma(z)$.

We now consider the special cases of (2.1) that occur in sieve theory. For $\kappa>0$, define $p_{\kappa}(u):=q(u, \kappa,-\kappa)$ and $q_{\kappa}(u):=q(u, \kappa, \kappa)$. The function $p_{-\kappa}(u)$ is the adjoint of the function $j_{\kappa}(u)$ (see $\left.\S 4\right)$ and $q_{\kappa}(u)$ and $p_{\kappa}(u)$ are 
used to determine some of the fundamental parameters of the combinatorial sieve. Specifically, the best sieve upper and lower bound functions yet known, $F_{\kappa}(u)$ and $f_{\kappa}(u)$ respectively, are the continuous solutions to the differentialdifference system

$$
\begin{aligned}
F(u) & =F_{0}(u), & & 0<u \leq \alpha_{\kappa}, \\
f(u) & =f_{0}(u), & & 0<u \leq \beta_{\kappa}, \\
\left(u^{\kappa} F(u)\right)^{\prime} & =\kappa u^{\kappa-1} f(u-1), & & u>\alpha_{\kappa}, \\
\left(u^{\kappa} f(u)\right)^{\prime} & =\kappa u^{\kappa-1} F(u-1), & & u>\beta_{\kappa},
\end{aligned}
$$

for given initial functions $F_{0}(u)$ and $f_{0}(u)$. The numbers $\alpha_{\kappa}$ and $\beta_{\kappa}$ are roots of equations involving $p_{\kappa}(u)$ and $q_{\kappa}(u)$ (see [I2, DH, DHR2]). For example, if $\kappa>1$ then $F_{0}(u):=1 / \sigma_{\kappa}(u), f_{0}(u):=0$, and $\alpha_{\kappa}$ and $\beta_{\kappa}$ are the solutions of (see [ILR])

$$
\left.\begin{array}{c}
\left.\begin{array}{c}
\Pi_{\kappa}(\alpha, \beta)=2 \\
\chi_{\kappa}(\alpha, \beta)=0
\end{array}\right\} \quad \text { if } \beta_{\kappa} \leq \alpha_{\kappa} \leq \beta_{\kappa}+1 \\
\Pi_{\kappa}(\alpha, \alpha-1)+\kappa \frac{p_{\kappa}(\alpha-1)}{(\alpha-1)^{\kappa-1}} \int_{\beta}^{(\gamma-1} \frac{t^{\kappa-1}}{\sigma_{\kappa}(t-1)} d t=2 \\
\chi_{\kappa}(\alpha, \alpha-1)-\kappa \frac{q_{\kappa}(\alpha-1)}{(\alpha-1)^{\kappa-1}} \int_{\beta}^{(\alpha-1} \frac{t^{\kappa-1}}{\sigma_{\kappa}(t-1)} d t=0
\end{array}\right\} \quad \text { if } \alpha_{\kappa} \geq \beta_{\kappa}+1,
$$

where for $u>0$ and $v>1$ we define

$$
\begin{aligned}
\Pi_{\kappa}(u, v) & :=\frac{u p_{\kappa}(u)}{\sigma_{\kappa}(u)}+\kappa \int_{v-1}^{u} \frac{p_{\kappa}(t+1)}{\sigma_{\kappa}(t)} d t \\
\chi_{\kappa}(u, v) & :=\frac{u q_{\kappa}(u)}{\sigma_{\kappa}(u)}-\kappa \int_{v-1}^{u} \frac{q_{\kappa}(t+1)}{\sigma_{\kappa}(t)} d t .
\end{aligned}
$$

Another, less complicated, example occurs for $1 / 2<\kappa<1$ where $\left(\alpha_{\kappa}, \beta_{\kappa}\right)=$ $\left(\rho_{\kappa}+2, \rho_{\kappa}+1\right)$ and $\rho_{\kappa}$ is defined as the largest root of $q_{\kappa}(u)$. It has not been shown that $\rho_{\kappa}$ is an increasing function of $\kappa$, although this is supported by the numerical evidence. So it is not surprising that the behavior of $\alpha_{\kappa}$ and $\beta_{\kappa}$ for $\kappa>1$ is even less well understood.

\section{THE FUNCTION $p(u, a, b)$}

We now look at a particular solution $p(u)=p(u, a, b)$ of equation (1.1), which is defined uniquely for all real $u, a$, and $b$ by the following six condi- 
tions:

$$
\begin{gathered}
u p^{\prime}(u)=-a p(u)-b p(u-1), \\
p(u)=\frac{e^{-b \gamma}}{\Gamma(1-a)} u^{-a} \quad(0<u \leq 1), \\
p(u)=0 \quad(u \leq 0), \\
p(u) \text { is continuous for } u>0 \text { when } a<1, \\
p(u, a, b)=\frac{d}{d u} p(u, a-1, b),
\end{gathered}
$$

$p(u)$ is left continuous everywhere and the derivatives in (3.1) and (3.5) are taken from the left at $u=0,1,2, \ldots$.

We need to prove that $p(u)$ exists and is unique. First assume $a<1$. The function $p(u)$ as defined by $(3.2)$ is integrable on $[0,1]$ and equation $(3.1)$ can be rewritten as $\left(u^{a} p(u)\right)^{\prime}=-b u^{a-1} p(u-1)$. This equation can be integrated forward a unit interval at a time, i.e.,

$$
p(u)=u^{-a}\left\{n^{a} p(n)-b \int_{n}^{u} t^{a-1} p(t-1) d t\right\} \quad(n<u \leq n+1, n \geq 1) .
$$

This establishes uniqueness and every condition except (3.5). To see that (3.5) holds, differentiate (3.1) and (3.2) to get $u p^{\prime \prime}(u)=-(a+1) p^{\prime}(u)-b p^{\prime}(u-1)$ and

$$
p^{\prime}(u)=\frac{e^{-b \gamma^{\prime}}}{\Gamma(1-(a+1))} u^{-(a+1)} \quad(0<u<1) .
$$

For the case $a \geq 1$, we make repeated use of this observation along with (3.5) and (3.6). If $n \geq 1$ with $a \leq n$, then

$$
p(u, a, b)=\frac{d^{n}}{d u^{n}} p(u, a-n, b)
$$

where the derivatives are taken from the left at $u=0,1,2, \ldots$.

In Theorem 1 below we discuss how the discontinuity at $u=0$ propagates itself forward to $u=1,2, \ldots,[a]$ when $a \geq 1$. We will also see that $p(u)$ is differentiable for all $u>[a]+1$. When $a \in \mathbf{Z}$, all of the discontinuities of $p(u)$, if any, are finite jump discontinuities, but if $a \notin \mathbf{Z}$ then $p(u)$ is unbounded to the right at any of its discontinuities. We need to point out that the Dickman function and its generalizations (see Example D) are usually defined to be right continuous at $u=0$. This differs from our definition but it will not cause any difficulties. Now we list a few special cases that follow easily from the definition of $p(u)$. 


$$
p(u, a, 0)=\frac{1}{\Gamma(1-a)} u^{-a} \quad(u>0),
$$

$$
\begin{aligned}
& p(u, 0, b)= \begin{cases}e^{-b \eta}, & 0<u \leq 1, \\
e^{-b \eta}(1-b \log u), & 1 \leq u \leq 2, \\
e^{-b \eta}\left(1-b \log u+b^{2} \int_{2}^{u} \frac{\log (t-1)}{t} d t\right), & 2 \leq u \leq 3,\end{cases} \\
& p(u, n, b)= \begin{cases}0, & u \leq 1, n \geq 1, \\
(-1)^{n}(n-1) ! b e^{-b u^{\prime}} u^{-n}, & 1<u \leq 2, n \geq 1 .\end{cases}
\end{aligned}
$$

For $a<1$, a general expression for $p(u, a, b)$ can be given in terms of the functions $K_{n}(u)=K_{n}(u, \lambda)$ defined for $\lambda>-1$ and $n \geq 0$ by $K_{n}(u)=0$ for $u \leq n, K_{0}(u)=u^{\lambda}$ for $u>0$, and

$$
K_{n}(u)=u^{\lambda} \int_{n}^{u} t^{-\lambda-1} K_{n-1}(t-1) d t \quad(u>n) .
$$

Now we can write

$$
p(u, a, b)=\frac{e^{-b_{\gamma}}}{\Gamma(1-a)} \sum_{n=0}^{\infty}(-b)^{n} K_{n}(u,-a) \quad(a<1) .
$$

The sum on the right is, of course, finite. For special cases of this expansion see [A, Bi, G, KTP, W]. In [G], Grupp uses a chain of power series to compute $K_{n}(u)$, whereas in [W] this function is computed using a single power series in the variable $(u-n) /(u-n+2)$.

Theorem 1. Let $b \neq 0$.

(i) For $a \notin \mathbf{Z}, p(u)$ is discontinuous if and only if $u=0,1, \ldots,[a]$ and at these values $p(u)$ has a finite left-sided limit and is unbounded from the right. Moreover, if $n \in\{0,1, \ldots,[a]\}$ then

$$
p(u) \sim \frac{(-b)^{n} e^{-b_{i}}}{n ! \Gamma(n-a+1)}(u-n)^{n-a} \quad\left(u \rightarrow n^{+}\right) .
$$

(ii) For $a \in \mathbf{Z}^{+}, p(u)$ is discontinuous if and only if $u=1,2, \ldots, a$, and these discontinuities are finite jumps.

Proof. (i) From the definition of $p(u)$ the statement is true for $a<1$. We shall use induction on $m=[a]+1$. For some $m \geq 1$, assume (i) is true for all $m-1<a<m$. Now

$$
\begin{aligned}
p(u, a+1, b) & =p^{\prime}(u, a, b) \\
& =-\frac{a}{u} p(u, a, b)-\frac{b}{u} p(u-1, a, b) \quad\left(u \notin \mathbf{Z}^{\geq 0}\right) .
\end{aligned}
$$

By the induction hypothesis the right-hand side of (3.10) is continuous for all $u$ except $u=0, \ldots, m$ and has a finite left-sided limit at each of these points. 
Thus all we need to show is that the estimate in (i) holds for all $0 \leq n \leq m$. The case $n=0$ holds automatically from (3.2). If $0<n<m$ then as $u \rightarrow n^{+}$ we have

$$
\begin{aligned}
p(u, a+1, b)= & \frac{-a(-b)^{n} e^{-b \gamma}}{u n ! \Gamma(n-a+1)}(u-n)^{n-a}(1+o(1)) \\
& -\frac{b(-b)^{n-1} e^{-b \gamma}}{u(n-1) ! \Gamma(n-a)}(u-1-(n-1))^{n-1-a}(1+o(1)) \\
= & \frac{(-b)^{n} e^{-b \gamma}}{n ! \Gamma(n-(a+1)+1)}(u-n)^{n-(a+1)}(1+o(1))
\end{aligned}
$$

and for $n=m$ the contribution from $-a p(u) / u$ is $O(1)$. This shows that $p(u, a+1, b)$ has discontinuities at $u=1, \ldots, m$ and thus completes the induction.

For (ii), start with (3.8) and (3.9) and proceed by induction using (3.10) as in the proof of (i) to see that the discontinuities can occur only at $u=1,2, \ldots, a$ and that they must be finite jump discontinuities. It is not obvious that these discontinuities actually exist. In the induction step when we take the left- and right-sided limits of the right-hand side of (3.10) at $u=n$ for some $n \in$ $\{1,2, \ldots, a\}$, it might happen that these two limits are equal; i.e., the jumps arising from each of the two terms on the right-hand side of (3.10) cancel each other out. We will now show that this cannot happen.

Define an array of rational functions $c_{m, n}=c_{m, n}(u, a, b)$ for $0 \leq n \leq m$ by the expansion

$$
p^{(m)}(u)=\sum_{n=0}^{m} c_{m, n} p(u-n)
$$

which is obtained by repeated differentiation of (3.10). Note that $c_{0,0}=1$, $c_{1,0}=-a / u, \quad c_{1,1}=-b / u, \quad c_{2,0}=a(a+1) / u^{2}, \quad c_{2,1}=b\left((a+1) / u^{2}+\right.$ $a / u(u-1))$, and $c_{2,2}=b^{2} / u(u-1)$. With $c_{m, n}^{\prime}=d c_{m, n}(u, a, b) / d u$, differentiating $p^{(m)}(u)$ gives us

$$
p^{(m+1)}(u)=\sum_{n=0}^{m}\left\{c_{m, n}^{\prime} p(u-n)-c_{m, n}\left(\frac{a}{u-n} p(u-n)+\frac{b}{u-n} p(u-n-1)\right)\right\} .
$$

Thus

$$
\begin{aligned}
& c_{m+1,0}=c_{m, 0}^{\prime}-\frac{a}{u} c_{m, 0} \Rightarrow c_{m, 0}=(-1)^{m} \frac{a(a+1) \cdots(a+m-1)}{u^{m}}, \\
& c_{m+1, m+1}=\frac{-b}{u-m} c_{m, m} \Rightarrow c_{m, m}=\frac{(-b)^{m}}{u(u-1) \cdots(u-m+1)},
\end{aligned}
$$

and

$$
c_{m+1, n}=c_{m, n}^{\prime}-\frac{a}{u-n} c_{m, n}-\frac{b}{u-n+1} c_{m, n-1} \quad(1 \leq n \leq m) .
$$


Now let $a=0$. We immediately get $c_{m, 0}=0$ for $m \geq 1$. Let $S_{m, n}$ denote the set of functions of the form

$$
(-1)^{m} b^{n} \sum d_{\mathrm{e}} u^{-e_{1}}(u-1)^{-c_{2}} \ldots(u-n+1)^{-e_{n}}
$$

where the coefficients $d_{\mathbf{e}}$ are nonnegative, not all zero, and independent of $b$ and $u$ and the sum is taken over all $\mathbf{e}=\left\langle e_{1}, \ldots, e_{n}\right\rangle$ such that $e_{i} \in \mathbf{Z}^{\geq 0}$ and $\sum_{i=1}^{n} e_{i}=m$. Take $S_{0,0}=\{1\}$ and $S_{m, 0}=\{0\}$ for $m \geq 1$. If $c_{m, n} \in S_{m, n}$ for all $0 \leq n \leq m$ then $c_{m, n}^{\prime} \in S_{m+1, n}$ and $(-b /(u-n+1)) c_{m, n-1} \in S_{m+1, n}$, and so (3.11) implies $c_{m+1, n} \in S_{m+1, n}$ since $S_{m+1, n}$ is additively closed. By induction $c_{m, n} \in S_{m, n}$, so $c_{m, n}$ is continuous and nonzero for $u>n-1$ when $n \geq 1$. Lastly, $p(u)=p(u, 0, b)$ is continuous except for a jump at $u=0$ and so

$$
p(u, m, b)=p^{(m)}(u)=\sum_{n=0}^{m} c_{m, n} p(u-n)
$$

along with $c_{m, n}(n) \neq 0$ shows us that $p(u, m, b)$ must have a jump discontinuity at $u=n$ for all $n \in\{1, \ldots, m\}$.

\section{THE FUNCTION $j_{\kappa}(u)$}

Now we would like to call attention to a subset of the $p$-family of functions which plays a role similar to that of the $Q_{n}$ in the $q$-family. It is the subset for which $a+b \in \mathbf{Z}$. We use the notation of Grupp and Richert [GR] by putting $j_{\kappa}(u):=p(u,-\kappa, \kappa)$ and also $j_{\kappa}^{(n)}(u):=p(u, n-\kappa, \kappa)$. For negative $n$ this is a slight abuse of notation, since $p(u, a-1, b)=\int_{0}^{u} p(t, a, b) d t$ is valid only for $a<1$ whereas differentiation is always valid (recall (3.5) and (3.6)). Therefore $j_{\kappa}^{(-1)}(u)=\int_{0}^{u} j_{\kappa}(t) d t$ holds only for $\kappa>-1$, etc.

The functions $j_{\kappa}(u)$ and $j_{\kappa}^{\prime}(u)$ for $\kappa>0$ have appeared frequently in sieve methods (with $\kappa$ denoting the "dimension") and in the study of incomplete sums of multiplicative functions. Also, the Dickman function is given by $\rho(u)=$ $e^{\gamma} j_{1}^{\prime}(u)$. Two of the more prominent notations for these functions that have appeared in the literature are $J_{\alpha}(u)=e^{\alpha \gamma \gamma} \Gamma(\alpha) j_{\alpha}(u)$ and $\tau_{\alpha}(u)=e^{\alpha \gamma} \Gamma(\alpha) j_{\alpha}^{\prime}(u)$ in [AO] and $\theta_{b}(u)=e^{b \gamma} \Gamma(1+b) j_{b}(u)$ and $\eta(u)=e^{b \gamma} \Gamma(b) j_{b}^{\prime}(u)$ in [dBvL].

A great deal is known about $j_{\kappa}$ and $j_{\kappa}^{\prime}$ for $\kappa>0$, some of which we summarize briefly (see $[\mathrm{AO}, \mathrm{GR}])$. Let $\kappa>0$. If $u>0$ then $j_{\kappa}(u)$ and $j_{\kappa}^{\prime}(u)$ are continuous and positive with $j_{\kappa}(u)$ increasing to 1 as $u \rightarrow \infty$. Furthermore, as $u \rightarrow \infty$ we have that

$$
j_{\kappa}(u)=1+O\left(e^{-u \log u}\right), \quad j_{\kappa}^{\prime}(u)=O\left(e^{-u \log u}\right)
$$

and also

$$
\int_{0}^{\infty} j_{\kappa}^{\prime}(u) d u=1
$$

For $\kappa>1, j_{\kappa}^{\prime}(u)$ has a unique stationary point at $u_{2}=u_{2}(\kappa)$, and for $\kappa>2$, two inflection points $u_{3}$ and $v_{3}$ with $1<u_{3}<u_{2}<u_{2}+1<v_{3}$. Also, $u_{2}$, 
$u_{3}$, and $v_{3}$ are increasing functions of $\kappa$ (see [GR]) with $\kappa-1 / 2<u_{2}<\kappa$ and

$$
\left\{\begin{array}{l}
\kappa-\sqrt{\kappa / 2}-1<u_{3}<\kappa-\sqrt{\kappa / 2} \\
\kappa+\sqrt{\kappa / 2}-1<v_{3}<\kappa+\sqrt{\kappa / 2}
\end{array}\right.
$$

Lastly, for $\kappa>1$ define $\mu_{\kappa}=\max j_{\kappa}^{\prime}(u)=j_{\kappa}^{\prime}\left(u_{2}\right)$; then $\mu_{\kappa}$ is a decreasing function of $\kappa$ (see [W]) with

$$
\mu_{\kappa} \sim 1 / \sqrt{\pi \kappa} \quad(\kappa \rightarrow \infty) .
$$

Another way of looking at $j_{\kappa}(u)$ for $\kappa>0$ is as a probability distribution function and at $j_{\kappa}^{\prime}(u)$ as the corresponding density function on $[0, \infty)$. This distribution has mean $\kappa$ and standard deviation $\sqrt{\kappa / 2}$, both of which follow from Theorem 2 below. In this context it is interesting to evaluate $j_{\kappa}$ at its mean. From [GR] we know $j_{\kappa}(\kappa)$ decreases to $1 / 2$ as $\kappa \rightarrow \infty$, and from [W] we have

$$
j_{\kappa}(\kappa)=\frac{1}{2}+1 / 9 \sqrt{\pi \kappa}+O\left(\kappa^{-3 / 2}\right) \quad(\kappa \geq 1) .
$$

We can give two simple models in which $j_{\kappa}(u)$ arises directly as a distribution function. Let $X_{1}, X_{2}, X_{3}, \ldots$ be independent random variables each uniformly distributed on $(0,1)$. By a generalization of a result of Chamayou (see [Ch] for $\kappa=1$ or [W]), if

$$
Z=X_{1}^{1 / \kappa}+\left(X_{1} X_{2}\right)^{1 / \kappa}+\left(X_{1} X_{2} X_{3}\right)^{1 / \kappa}+\cdots
$$

then

$$
\operatorname{Pr}\{Z \leq u\}=j_{\kappa}(u) .
$$

Second, it follows from a result of Hensley [He] that if

$$
Z_{n}=e^{-(n / \kappa) X_{1}}+e^{-(n / \kappa) X_{2}}+\cdots+e^{-(n / \kappa) X_{n}}
$$

then

$$
\lim _{n \rightarrow \infty} \operatorname{Pr}\left\{Z_{n} \leq u\right\}=j_{\kappa}(u) .
$$

\section{The Laplace TRANSFORM OF $p(u)$}

Note that $Q_{n-1}(u, \kappa)$ is the adjoint of $j_{\kappa}^{(n)}(u)$ for $n \geq 1$. One of the reasons that $Q_{n}$ stands out in the $q$-family is that it is entire. Similarly, we will see in the following theorem that $j_{\kappa}^{(n)}$ stands out in the $p$-family because its Laplace transform, when it exists, is entire for $n>1$ and meromorphic for $n \leq 0$.

\section{Theorem 2.}

(i) For $a<1, a+b \notin \mathbf{Z}^{+}$, and $\mathfrak{R} s>0$ we have

$$
\int_{0}^{\infty} e^{-s t} p(t) d t=s^{a+b-1} e^{-b \operatorname{Ein}(s)} .
$$

(ii) For $n \geq 1, \kappa>n-1$, and all $s \in \mathrm{C}$ we have

$$
\int_{0}^{\infty} e^{-s t} j_{\kappa}^{(n)}(t) d t=s^{n-1} e^{-\kappa \operatorname{Ein}(s)}
$$


(iii) For $n \geq 0, \kappa>-n-1$, and all $s \in \mathbf{C}$ we have

$$
\begin{aligned}
\int_{0}^{\infty} e^{-s t}\left\{j_{\kappa}^{(-n)}(t)-\frac{(-1)^{n}}{n !} Q_{n}(-t,-\kappa)\right\} d t \\
=s^{-n-1}\left\{e^{-\kappa \operatorname{Ein}(s)}-\sum_{m=0}^{n} Q_{m}(0,-\kappa) \frac{(-1)^{m}}{m !} s^{m}\right\} .
\end{aligned}
$$

Proof. Part (i) can be found in [dBvL, LF, U] and special cases of either (i) or (ii) are given in [AO, GM1, GR, HR, He, I1]. We shall sketch proofs of (i) and (ii) that do not depend on knowing any asymptotic information about $p(u)$ beforehand.

First note that except for the region of convergence, (ii) is a restatement of (i) cast in the $j$-notation with $(a, b)=(n-\kappa, \kappa)$. We begin with formal arguments which lead to the Laplace transform in (i). Write $F(s)=$ $\int_{0}^{\infty} e^{-s t} p(t) d t$. Since $p(u)=0$ for all $u<0$ and $p(u)$ satisfies (1.1), we have that $p(u)=C_{0} u^{-a}$ for all $u \in(0,1)$ and some constant $C_{0}$. Thus $a<1$ implies $\lim _{u \rightarrow 0+} u p(u)=0$. Recall that if $f(t)$ is continuous, $\mathscr{L}\{f(t)\}=F(s)$, and $\mathscr{L}\left\{f^{\prime}(t)\right\}$ exists, then

$$
\int_{0}^{\infty} e^{-s t} f^{\prime}(t) d t=s F(s)-f(0+) .
$$

So writing $(1.1)$ in the form

$$
(u p(u))^{\prime}=(1-a) p(u)-b p(u-1)
$$

and using standard Laplace transform manipulations, we see that (5.2) gets mapped into the ordinary differential equation $s F^{\prime}(s)=\left(a-1+b e^{-s}\right) F(s)$. Solving this equation, we get $F(s)=C_{1} s^{a+b-1} e^{-b \operatorname{Ein}(s)}$ for some constant $C_{1}$. The relationship between $C_{0}$ and $C_{1}$ is found by noting the behavior of $F(s)$ as $s \rightarrow+\infty$. Equations (1.4) and (1.5) immediately imply that $F(s) \sim C_{1} e^{-b \gamma} s^{a-1}$ as $s \rightarrow+\infty$. From the definition of $F(s)$ along with $p(u)=C_{0} u^{-a}$ we get

$$
F(s) \sim C_{0} \int_{0}^{\infty} e^{-s t} t^{-a} d t=C_{0} \Gamma(1-a) s^{a-1} \quad(s \rightarrow+\infty)
$$

so that

$$
C_{0}=\left(e^{-b \gamma} / \Gamma(1-a)\right) C_{1} .
$$

We have chosen $C_{1}=1$, a natural choice for sieve methods (see $\S 8$ ).

To prove (i) and (ii) we use a result of Doetsch [Do2] which gives a sufficient condition for a function to be representable as a Laplace transform.

Lemma (Doetsch [Do2, p. 187]). When $F(s)=F(x+i y)$ is analytic in the halfplane $x>x_{1}$; when $F(s)$ converges, in every half-plane $x \geq x_{1}+\delta>x_{1}$, toward 0 , when $s$ tends two-dimensionally toward $\infty$, i.e., in any direction within the half-plane; and when

$$
\int_{-\infty}^{\infty}|F(x+i y)| d y<\infty
$$


for every $x>x_{1}$; then it follows that $F(s)$ may be represented as the Laplace transform of the original function

$$
f(t)=\frac{1}{2 \pi i} \int_{x-i \infty}^{x+i \infty} e^{t s} F(s) d s \quad\left(x>x_{1}\right),
$$

the integral being independent of the choice of $x$ in $x>x_{1}$. For $t<0$, the integral in (5.4) yields the value zero. The function $f(t)$ is continuous in $-\infty<t<\infty$.

First consider $a<0$. Let $F(s)=s^{a+b-1} e^{-b \operatorname{Ein}(s)}$, which is analytic on $\left\{s: \mathfrak{R} s>x_{1}\right\}$, where $x_{1}=0$ if $a+b \notin \mathbf{Z}^{+}$and $x_{1}=-\infty$ if $a+b \in \mathbf{Z}^{+}$. By (1.4) we have $|F(s)|=e^{-b \gamma}|s|^{a-1} e^{-b \Re E_{1}(s)}$ for $|\arg s|<\pi$ and because of (1.5) it is easy to see that $F(s) \rightarrow 0$ as $s$ tends two-dimensionally toward $\infty$ in any proper right half-plane. For fixed $x>x_{1},|F(x+i y)| \sim e^{-b \gamma}|x+i y|^{a-1}$ as $y \rightarrow \pm \infty$ and hence the integral in (5.3) converges when $a<0$. Thus $f(t)$, as given by (5.4), is continuous on $\mathbf{R}$ and 0 for $t<0$. It is easily shown that $f$ satisfies $(5.2)$ on $\mathbf{R}$. Since $p(u)$ is the unique continuous solution of (5.2) which satisfies (3.2), we have proved (i) and (ii) for $a<0$.

For the case $0 \leq a<1$, note that (3.2) implies that the integral defining the Laplace transform of $p(t)$ converges at $t=0$. Also, (3.1) and (3.5) give

$$
\begin{aligned}
\int_{0}^{\infty} & e^{-s t} p(t, a, b) d t \\
\quad & =-(a-1) \int_{0}^{\infty} e^{-s t} \frac{p(t, a-1, b)}{t} d t-b \int_{0}^{\infty} e^{-s t} \frac{p(t-1, a-1, b)}{t} d t
\end{aligned}
$$

which implies convergence at $t=\infty$. Since $p(t, a, b)=p^{\prime}(t, a-1, b)$ for all $t>0$ and $p(0+, a-1, b)=0$, equation (5.1) finishes the proof of (i) and (ii) for all $a<1$.

We now turn to (iii). The right-hand side of the equation in (iii) is entire because of (2.6) with $u=0$. Also, for $R s>0$ the Laplace transform of $\left((-1)^{n} / n !\right) Q_{n}(-t,-\kappa)$ is easily seen to be $\sum_{m=0}^{n} Q_{m}(0,-\kappa)\left((-1)^{m} / m !\right) s^{m-n-1}$ by using (2.7) with $v=0$. Combining this with (i), we see that (iii) holds for $\mathfrak{R} s>0$. Actually, (iii) can be shown to be valid for all $s \in \mathbf{C}$ when $\kappa>0$ by starting with (ii), $n=1$, and repeatedly integrating $j_{\kappa}^{\prime}(t)$ from $t=u$ to $\infty$; e.g., $1-j_{\kappa}(u)=\int_{u}^{\infty} j_{\kappa}^{\prime}(t) d t$ for all $u \in \mathbf{R}$ when $\kappa>0$. But to prove (iii) for $\kappa>-n-1$ we shall use some asymptotic information about $j_{\kappa}^{(-n)}(t)$ found in [LF, p. 139, $\tau_{0}=\kappa+n$ ]. In our notation, Levin and Fainleib showed that there exists a polynomial $R_{n}(t)$ of degree $n$ such that $j_{\kappa}^{(-n)}(t)=$ $R_{n}(t)+O\left(e^{-t \log t}\right)$ as $t \rightarrow \infty$. Thus for $\kappa>-n-1$ the Laplace transform of $j_{\kappa}^{(-n)}(t)-R_{n}(t)$ will converge for all $s \in \mathbf{C}$. But we see from the equation in (iii) that $\left((-1)^{n} / n !\right) Q_{n}(-t,-\kappa)$ is the unique polynomial which makes the right-hand side of this equation entire. Thus $R_{n}(t)=\left((-1)^{n} / n !\right) Q_{n}(-t,-\kappa)$ and (iii) holds for all $s \in \mathrm{C}$. In [LF] only the leading coefficient of $R_{n}(t)$ was found; here we have determined $R_{n}(t)$ explicitly. 
One application of Theorem 2 is in computing $\partial j_{\kappa}^{(n)}(u) / \partial \kappa$. From (i) we know that (ii) is valid for all integers $n$ and all $\kappa>n-1$ when $\Re s>0$. Using (5.4) we can invert the Laplace transform in (ii) with $n \in \mathbf{Z}$ and $\kappa>n-1$, and after formally differentiating under the integral sign we get

$$
\frac{\partial}{\partial \kappa} j_{\kappa}^{(n)}(t)=\frac{-1}{2 \pi i} \int_{x-i \infty}^{x+i \infty} e^{t s} s^{n-1} e^{-\kappa \operatorname{Ein}(s)} \operatorname{Ein}(s) d s \quad(x>0) .
$$

The integral on the right is absolutely convergent for $n-1-\kappa<-1$ (see (1.4)) and so $\partial j_{\kappa}^{(n)}(t) / \partial \kappa$ exists for $\kappa>n$ and $t>0$. Using a change of variables on $\int_{0}^{s}\left(1-e^{t}\right) / t d t$, we see that $\operatorname{Ein}(s) / s$ can be represented as a Laplace transform for all $s \in \mathbf{C}$, namely

$$
\frac{\operatorname{Ein}(s)}{s}=\int_{0}^{1} e^{-s t} \log (1 / t) d t
$$

Differentiating the Laplace transform in Theorem 2(ii) with respect to $\kappa$, we have

$$
\int_{0}^{\infty} e^{-s t} \frac{\partial}{\partial \kappa} j_{\kappa}^{(n)}(t) d t=-\frac{\operatorname{Ein}(s)}{s} s^{n} e^{-\kappa \operatorname{Ein}(s)} \quad(\kappa>n, \mathfrak{R} s>0) .
$$

Using the convolution theorem for Laplace transforms on Theorem 2(ii) and (5.5), we get for $\kappa>n$ and $u>0$

$$
\begin{aligned}
\frac{\partial}{\partial \kappa} j_{\kappa}^{(n)}(u) & =-\int_{0}^{1} j_{\kappa}^{(n+1)}(u-t) \log (1 / t) d t \\
& =\int_{0}^{1}\left\{j_{\kappa}^{(n)}(u-t)-j_{\kappa}^{(n)}(u)\right\} \frac{d t}{t}
\end{aligned}
$$

The second equation follows from the first using integration by parts. These two equations are put to a great deal of use in [GR] and the case $n=1$ can also be found in [He]. Another convolution formula that is readily apparent from Theorem $2(\mathrm{i})$ is

$$
p(u, a, b) * p(u, \alpha, \beta)=p(u, a+\alpha-1, b+\beta) \quad(a<1, \alpha<1) .
$$

This is noted in [U] and special cases are used in [AO] and [HR]. The case $(\alpha, \beta)=(1-x, 0)($ see $(3.7))$ gives

$$
p(u, a, b) * u^{x-1} / \Gamma(x)=p(u, a-x, b) \quad(a<1, x>0),
$$

which generalizes (3.5) to "fractional derivatives".

\section{THE ASYMPTOTIC BEHAVIOR OF $p(u)$}

In the proof of the Laplace transform given in Theorem 2(i) we avoided the use of asymptotic or even $O$-information about $p(u)$. We now use this result to give an explicit asymptotic expansion of $p(u)$ as $u \rightarrow \infty$ when $a+b \notin \mathbf{Z}$. 


\section{Theorem 3.}

(i) For $a+b \notin \mathbf{Z}$ and $c \in \mathbf{R}$ we have

$$
p(u, a, b) \sim \sum_{n=0}^{\infty}(-1)^{n} \frac{Q_{n}(c,-b)}{n ! \Gamma(1-a-b-n)}(u+c)^{-a-b-n} \quad(u \rightarrow \infty) .
$$

(ii) For all $\kappa \in \mathbf{R}$ and $n \geq 0$ we have

$$
j_{\kappa}^{(n+1)}(u)=O\left(e^{-u \log u}\right) \quad(u \rightarrow \infty)
$$

and

$$
j_{\kappa}^{(-n)}(u)=\left((-1)^{n} / n !\right) Q_{n}(-u,-\kappa)+O\left(e^{-u \log u}\right) \quad(u \rightarrow \infty) .
$$

(iii) Define the function $\xi(u)$ for all $u>0$ as the unique real solution of $\left(e^{\xi(u)}-1\right) / \xi(u)=u$. For $\kappa>0, n \geq 0$, and $\xi=\xi(u / \kappa)$ we have

$$
j_{\kappa}^{(n+1)}(u) \sim \frac{(-1)^{n}}{\sqrt{2 \pi u}} \xi^{n} e^{-u \xi-\kappa \operatorname{Ein}(-\xi)}\left(1-\frac{1-\kappa / u}{\xi}\right)^{-1 / 2} \quad(u \rightarrow \infty) .
$$

Proof. (i) The case $(a, b, c)=(0, \delta, 0)$ for $0<\delta<1$ can be found in [GM1]. We shall expand on their proof. Note the similarity between the expansion in (i) and the asymptotic expansion for $q(u, a, b)$ given in Theorem 11 below.

We first show that if (i) is valid for $c=0$, this implies that (i) must also be valid for all $c \in \mathbf{R}$. The generalized Euler transformation (see [O] for $\lambda=0$ ) states that if

$$
\beta_{n}=\sum_{m=0}^{n}\left(\begin{array}{c}
n+\lambda \\
m+\lambda
\end{array}\right) \alpha_{m} c^{n-m}
$$

then formally we have

$$
\sum_{n=0}^{\infty} \frac{\alpha_{n}}{u^{\lambda+n+1}}=\sum_{n=0}^{\infty} \frac{\beta_{n}}{(u+c)^{\lambda+n+1}}
$$

where as usual

$$
\left(\begin{array}{c}
z \\
w
\end{array}\right):=\frac{\Gamma(z+1)}{\Gamma(w+1) \Gamma(z-w+1)} .
$$

The proof is a straightforward application of the binomial expansion. Applying (6.1) to the series in (i) with $c=0$ and $\lambda=a+b-1$ and using the reflection formula $\Gamma(z) \Gamma(1-z)=\pi \csc (\pi z)$ we get

$$
\begin{aligned}
\beta_{n} & =\sum_{m=0}^{n} \frac{\Gamma(n+a+b)}{(n-m) ! \Gamma(m+a+b)} \cdot \frac{(-1)^{m} Q_{m}(0,-b)}{m ! \Gamma(1-a-b-m)} c^{n-m} \\
& =\frac{1}{n !} \sum_{m=0}^{n}\left(\begin{array}{l}
n \\
m
\end{array}\right) \frac{(-1)^{n}}{\Gamma(1-a-b-n)} \cdot \frac{\sin \pi(a+b+m)}{\sin \pi(a+b+n)} Q_{m}(0,-b) c^{n-m} \\
& =\frac{(-1)^{n} Q_{n}(c,-b)}{n ! \Gamma(1-a-b-n)} .
\end{aligned}
$$

The iast equality follows from (2.7). Thus we need only prove part (i) for $c=0$. 
First assume $a+b \in \mathbf{Z}, c=0$, and $a<1$. Let $N=\max (0,[-a-b])$ and $\alpha_{n}=\left((-1)^{n} / n !\right) Q_{n}(0,-b)$. For $\mathfrak{R s}>0$, Theorem 2(i) and (2.6) give

$$
\begin{aligned}
\int_{0}^{\infty}\left\{p(t)-\sum_{n=0}^{N-1} \frac{\alpha_{n} t^{-a-b-n}}{\Gamma(1-a-b-n)}\right\} d t & =s^{a+b-1} e^{-b \operatorname{Ein}(s)}-\sum_{n=0}^{N-1} \alpha_{n} s^{a+b-1+n} \\
& =\sum_{n=N}^{\infty} \alpha_{n} s^{a+b-1+n} .
\end{aligned}
$$

Since $a+b-1+N>-1$, the lemma below immediately gives (i) with $c=0$ and $a<1$.

Lemma (Doetsch [Do1, p. 150]). Let $f(t)$ have Laplace transform $F(s)$ and let $F(s)$ be given by the absolutely convergent series expansion

$$
F(s)=\sum_{n=0}^{\infty} a_{n} s^{\lambda_{n}} \quad\left(-1<\mathfrak{R} \lambda_{0}<\mathfrak{R} \lambda_{1}<\cdots\right)
$$

in some sector $\{z:|\arg z| \leq \theta, z \neq 0\}$ with $\pi / 2<\theta<\pi$. Then

$$
f(t) \sim \sum_{n=0}^{\infty} \frac{a_{n}}{\Gamma\left(-\lambda_{n}\right)} t^{-\lambda_{n}-1} \quad(t \rightarrow \infty) .
$$

Now consider $a+b \notin \mathbf{Z}, c=0$, and $a \geq 1$. For fixed $b$, if (i) is true for $a=a_{0}$, then for sufficiently large $u(3.5)$ gives $p\left(u, a_{0}+1, b\right)=p^{\prime}\left(u, a_{0}, b\right)$. Term-by-term differentiation of the asymptotic series in (i) with $a=a_{0}$ gives the series back with $a=a_{0}+1$. Differentiating the asymptotic series in this situation is valid because

$$
p\left(u, a_{0}+1, b\right)=(-1 / u)\left(a_{0} p\left(u, a_{0}, b\right)+b p\left(u-1, a_{0}, b\right)\right) \quad\left(u>u_{0}\right)
$$

implies $p\left(u, a_{0}+1, b\right)$ has an asymptotic expansion in $\left\{u^{-\left(a_{0}+1\right)-b-n}\right\}_{n=0}^{\infty}$. This implies that the expansion can be found by term-by-term differentiation (see [BH, Theorem 1.7.7, p. 32]). Thus induction on [a] proves (i) for all $a \geq 1$.

(ii) This follows from [LF, (1.3.4) and (1.3.6)]. The identification of the polynomial asymptote of $j_{\kappa}^{(-n)}(u)$ for $n \geq 0$ comes from Theorem 2(iii), its proof, and the fact that

$$
\frac{d}{d u} \frac{(-1)^{n}}{n !} Q_{n}(-u,-\kappa)=\frac{(-1)^{n-1}}{(n-1) !} Q_{n-1}(-u,-\kappa)
$$

(iii) The asymptotic formula in (iii) was proved first by de Bruijn [dB3] for the Dickman function $\rho(u)$, i.e., for the case $n=0$ and $\kappa=1$. He used a result on Volterra integral equations [dB2] and two applications of the saddle point method. Later, other proofs were given by van Ouwerkerk-Dijkers and Nuis [vODN], who used the saddle point method directly on the inverse Laplace transform of $\rho(u)$, and by Canfield [Ca], who used generating functions and 
the circle method. Hensley [He] gave a uniform result for the case $n=0$ using the Berry-Esseen theorem of probability theory. He showed that

$$
j_{\kappa}^{\prime}(u)=(2 \pi u)^{-1 / 2}\left(1-\frac{1-\kappa / u}{\xi}\right)^{-1 / 2} e^{-u \xi-\kappa \operatorname{Ein}(-\xi)}\left\{1+O\left((u+\kappa)^{-1 / 3}\right)\right\}
$$

uniformly in $\kappa \geq \varepsilon>0$ and $u \geq 1$.

To prove (iii) we start with Hensley's result on $j_{\kappa}^{\prime}(u)$ and use induction on $n$. The asymptotic formula (iii) will follow from

$$
j_{\kappa}^{(n+2)}(u) / j_{\kappa}^{(n+1)}(u) \sim-\xi(u / \kappa) \quad(u \rightarrow \infty, n \geq 0) .
$$

From [vODN] we have

$$
\xi=\log u+\log \log u+\frac{\log \log u}{\log u}-\frac{1}{2}\left(\frac{\log \log u}{\log u}\right)^{2}+O\left(\frac{\log \log u}{\log ^{2} u}\right) \quad(u \rightarrow \infty)
$$

and a small calculation shows

$$
\begin{aligned}
& \xi(u / \kappa)=\log u+\log \log u-\log \kappa \\
& \quad+\frac{\log \log u}{\log u}-\frac{\log \kappa}{\log u}-\frac{1}{2}\left(\frac{\log \log u}{\log u}\right)^{2}+O\left(\frac{\log \log u}{\log ^{2} u}\right) \quad(u \rightarrow \infty) .
\end{aligned}
$$

Assume (iii) holds for some $n \geq 0$. From (3.1) with $(a, b)=(n+1-\kappa, \kappa)$ and $u$ sufficiently large we have

$$
\begin{aligned}
-u j_{\kappa}^{(n+2)}(u) / j_{\kappa}^{(n+1)}(u) & =\kappa j_{\kappa}^{(n+1)}(u-1) / j_{\kappa}^{(n+1)}(u)-\kappa+n+1 \\
& \sim \kappa e^{g(u)-g(u-1)}-\kappa+n+1 \quad(u \rightarrow \infty)
\end{aligned}
$$

where $g(u):=u \xi(u / \kappa)+\kappa \operatorname{Ein}(-\xi(u / \kappa))$. Note that $g(u)-g(u-1)=g^{\prime}\left(u^{*}\right)$ for some $u^{*} \in(u-1, u)$ and

$$
g^{\prime}(u)=\xi(u / \kappa)+u \frac{d}{d u} \xi(u / \kappa)+\kappa \frac{e^{\xi(u / \kappa)}-1}{\xi(u / \kappa)} \frac{d}{d u}(-\xi(u / \kappa))=\xi(u / \kappa) .
$$

Thus

$$
e^{g(u)-g(u-1)}=e^{\xi\left(u^{*} / \kappa\right)}=\frac{u^{*}}{\kappa} \xi\left(u^{*} / \kappa\right)+1 \sim \frac{u}{\kappa} \xi(u / \kappa) \quad(u \rightarrow \infty)
$$

and combining this with (6.3) finishes the proof of (iii).

We now give an approximation to $j_{\kappa}^{(n+1)}(u)$ which is simpler than Theorem 3(iii). From (5.5) it is not hard to deduce that

$$
-\operatorname{Ein}(-z)=z e^{z} \int_{0}^{1} e^{-z t} \log \frac{1}{1-t} d t \quad(z \in \mathbf{C}),
$$

which implies, after expanding $-\log (1-t)$ in a power series,

$$
-\operatorname{Ein}(-z) \sim \frac{e^{z}}{z}\left\{1+\frac{1 !}{z}+\frac{2 !}{z^{2}}+\cdots\right\} \quad(z \rightarrow \infty) .
$$


Combining this with $(6.2)$ we get

$$
\begin{aligned}
-\kappa \operatorname{Ein}(-\xi(u / \kappa)) & =\kappa \frac{e^{\xi(u / \kappa)}}{\xi(u / \kappa)}\left(1+\xi^{-1}(u / \kappa)+O\left(\xi^{-2}(u / \kappa)\right)\right) \\
& =u\left(1+\xi^{-1}(u / \kappa)+O\left(\xi^{-2}(u / \kappa)\right)\right) \\
& =u\left(1+\frac{1}{\log u}+O\left(\frac{\log \log u}{\log ^{2} u}\right)\right)
\end{aligned}
$$

Substituting this along with (6.2) into the asymptotic estimate in Theorem 3(iii) gives

$$
\begin{array}{r}
j_{\kappa}^{(n+1)}(u)=(-1)^{n} \exp \left\{-u\left(\log u+\log \log u-(1+\log \kappa)+\frac{\log \log u}{\log u}\right.\right. \\
\left.\left.-\frac{1+\log \kappa}{\log u}-\frac{1}{2}\left(\frac{\log \log u}{\log u}\right)^{2}+O\left(\frac{\log \log u}{\log ^{2} u}\right)\right)\right\}
\end{array}
$$

for $\kappa>0$ and sufficiently large $u$.

\section{THE ADJOINT RELATION}

We now use the asymptotic information found in Theorem 3 to evaluate the constant $C$ in the adjoint equation (1.3).

Theorem 4. If $a, b \in \mathbf{R}$ then

$$
u p(u) q(u)-b \int_{u-1}^{u} p(t) q(t+1) d t=\frac{1}{\Gamma(1-a-b)}
$$

for all $u>\max ([a], 0)$ or for all $u \in \mathbf{R}$ if $a<1$ and $a+b \in \mathbf{Z}^{+}$.

Proof. As was mentioned in the introduction, the adjoint relation (1.3) is well known (e.g., see [12]). What is new in Theorem 4 is the evaluation of the constant in the general case. For completeness, we start by proving (1.3). Let $f(u)$ denote the left-hand side of $(7.1)$. Whenever $p(u)$ is differentiable then $f(u)$ is differentiable and

$$
\begin{aligned}
f^{\prime}(u)= & (u q(u))^{\prime} p(u)+u p^{\prime}(u) q(u)-b p(u) q(u+1)+b p(u-1) q(u) \\
= & (a q(u)+b q(u+1)) p(u)+(-a p(u)-b p(u-1)) q(u) \\
& -b p(u) q(u+1)+b p(u-1) q(u) \\
= & 0
\end{aligned}
$$

Therefore $f(u)$ is constant on any interval of differentiability.

First assume $a+b \in \mathbf{Z}^{+}$and $a<1$. We know that $q$ is a polynomial and $u p(u) \rightarrow 0$ as $u \rightarrow 0^{+}$so that $f(u)$ is continuous for all $u \in \mathbf{R}$ and differentiable except possibly at $u=0,1$. From the preceding argument, $f(u)$ is constant for $u<0, u \in(0,1)$, and $u>1$ and the continuity of $f(u)$ tells us it is constant for all $u \in \mathbf{R}$. By evaluating $f(u)$ for $u<0$ we easily find this constant to be 0 . Now consider the general case. Let $u_{a}=\max ([a], 0)$. 
By Theorem 1, $f$ is continuous on $\left(u_{a}, \infty\right)$ and also differentiable on this interval except possibly at $u_{a}+1$. As before, this implies $f$ is constant on $\left(u_{a}, \infty\right)$, which we evaluate by examining $\lim _{u \rightarrow \infty} f(u)$. For $a+b \in \mathbf{Z}^{+}$recall that $p(u)=O\left(e^{-u}\right)$ and $q$ is a polynomial and so $\lim _{u \rightarrow \infty} f(u)=0$. For $a+b \notin \mathbf{Z}^{+}$, Theorem 3(i) and (2.3) imply $u p(u) q(u) \sim 1 / \Gamma(1-a-b)$ and

$$
\left|\int_{u-1}^{u} p(t) q(t+1) d t\right| \leq \max _{u-1 \leq t \leq u}|p(t) q(t+1)| \ll \frac{1}{u} \text {. }
$$

Thus $\lim _{u \rightarrow \infty} f(u)=1 / \Gamma(1-a-b)$ and this theorem is proved.

In the case $a+b \in \mathbf{Z}^{+}$we can rewrite (7.1) as

$$
u j_{\kappa}^{(n)}(u) Q_{n-1}(u, \kappa)-\kappa \int_{u-1}^{u} j_{\kappa}^{(n)}(t) Q_{n-1}(t+1, \kappa) d t=0
$$

for $u>[n-\kappa]$ and $n \geq 1$. As we discussed in the introduction, (7.2) can be used to gain information about the zeros of $j_{\kappa}^{(n)}(u)$ when we know the zeros of $Q_{n-1}(u, \kappa)$. Here is a specific example with $n=3$. Recall that $Q_{2}(u, \kappa)=$ $(u-\kappa)^{2}-\kappa / 2$ and so the roots are $\kappa \pm \sqrt{\kappa / 2}$. Setting $u$ in (7.2) to each of these roots in turn, for $\kappa>2$, gives the estimates in (4.1). We now present an "oscillation theorem" which is a more gradiose use of this technique. In Theorem 1 we saw that the value of $a$ governs the number and type of initial discontinuities of $p(u)$. Theorems 2 and 3 told us that the value of $a+b$ governs the asymptotic behavior of $p(u)$. Now we shall see that $b \quad(=\kappa$ in the $j$-notation), specifically its sign, governs how $p(u)$ behaves with respect to its polynomial asymptote when $a+b \in \mathbf{Z}$. The following result with $n=0$, in the context of Example $C$ (see $\S 8$ ), was recently used by Maier [M] in the study of the distribution of primes in short intervals.

\section{Theorem 5.}

(i) Let $n \geq 0$ and define

$$
J_{n}(u):=(-1)^{n+1}\left\{j_{\kappa}^{(-n)}(u)-\left((-1)^{n} / n !\right) Q_{n}(-u,-\kappa)\right\} .
$$

If $\kappa>0$ then $J_{n}(u)$ is positive for all $u>0$. If $\kappa<0$ then $J_{n}(u)$ has a zero in every interval $(\alpha, \alpha+1)$ for $\alpha>\max ([-n-\kappa], 0)$.

(ii) Similarly, let $n \geq 1$. If $\kappa>0$ then $(-1)^{n+1} j_{\kappa}^{(n)}(u)$ is positive for all sufficiently large $u$. If $\kappa<0$ then it has a zero in every interval $(\alpha, \alpha+1)$ for $\alpha>[n-\kappa]$ when $\kappa<0$.

Proof. (i) First assume $\kappa>0$. Since $a=-n-\kappa<0$, we know that $J_{n}(u)$ is continuous for all $u \in \mathbf{R}$. Now, upon integrating we get

$$
\begin{aligned}
\int_{0}^{u} J_{n}(t) d t & =(-1)^{n+1}\left\{j_{\kappa}^{(-n-1)}(u)-\left(\frac{(-1)^{n+1}}{(n+1) !} Q_{n+1}(-u,-\kappa)\right.\right. \\
& =-J_{n+1}(u)+\frac{1}{(n+1) !} Q_{n+1}(0,-\kappa) .
\end{aligned}
$$


By Theorem 2(iii) with $s=0$ we see that $\int_{0}^{\infty} J_{n}(t) d t=Q_{n+1}(0,-\kappa) /(n+1)$ !, which implies $J_{n+1}(u)=\int_{u}^{\infty} J_{n}(t) d t$. Since $J_{0}(u)=1-j_{\kappa}(u)>0$ for $u>0$ we get $J_{n}(u)>0$ for $u>0$ by induction.

Now assume $\kappa<0$. First note the fact that if $S \subseteq \mathbf{R}, \tau \neq 0$, and $f(u)=$ $p(u / \tau)$ then $p(u)$ satisfies (1.1) for all $u \in S$ if and only if $u f^{\prime}(u)=-a f(u)-$ $b f(u-\tau)$ for all $u \in \tau S$. Since $q(u)=Q_{n}(u,-\kappa)$ satisfies $(1.2)$ with $(a, b)=$ $(n+1+\kappa,-\kappa)$ for all $u \in \mathbf{R}, p(u)=Q(-u,-\kappa)(\tau=-1)$ satisfies (1.1) with $(a, b)=(-n-\kappa, \kappa)$. Thus $Q_{n}(-u,-\kappa)$ is adjoint to $q(u,-n-\kappa, \kappa)$ and so (1.3) implies for $u>0$

$u Q_{n}(-u,-\kappa) q(u,-n-\kappa, \kappa)-\kappa \int_{u-1}^{u} Q_{n}(-t,-\kappa) q(t+1,-n-\kappa, \kappa) d t=(-1)^{n}$.

The constant was determined by using the asymptotic estimates for $Q_{n}(-u,-\kappa)$ and $q(u,-n-\kappa, \kappa)$. Multiplying (7.3) by $(-1)^{n} / n$ ! and subtracting it from (7.1) with $(a, b)=(-n-\kappa, \kappa)$ gives

$$
\begin{array}{r}
u J_{n}(u) q(u,-n-\kappa, \kappa)=\kappa \int_{u-1}^{u} J_{n}(t) q(t+1,-n-\kappa, \kappa) d t \\
\quad(u>\max ([-n-\kappa], 0)) .
\end{array}
$$

Since $\kappa<0,(2.5)$ implies $q(u,-n-\kappa, \kappa)>0$ for all $u>0$. For $\alpha>$ $\max ([-n-\kappa], 0)), J_{n}(u)$ is continuous in $[\alpha, \infty)$. Thus evaluating (7.4) at $u=\alpha+1$, we immediately find that $J_{n}(u)$ must have a zero in $(\alpha, \alpha+1)$ since $\kappa<0$. Otherwise, if $J_{n}(u)$ is of one sign on $(\alpha, \alpha+1)$, then $\kappa<0$ implies that the left and right sides of (7.4) are of different signs.

(ii) Positivity follows from Theorem 3(iii) and the oscillations around zero follow from (7.2) as in (i).

\section{Applications of $p(u, a, b)$ IN NUMBeR theORY}

Functions of the type $p(u, a, b)$ occur in various number theoretic context; in this section we survey some of these applications. The first three applications are taken from the theory of sieves. Let $\mathscr{A}$ be a finite sequence of integers and let $\mathscr{P}$ be a set of primes. The sifting functions $S(\mathscr{A}, \mathscr{P}, z)$ is the number of elements of $\mathscr{A}$ that remain after "sifting" $\mathscr{A}$ by all the primes in $\mathscr{P}$ that are less than $z$. More precisely,

$$
S(\mathscr{A}, \mathscr{P}, z):=\#\{a \in \mathscr{A}:(a, P(z))=1\}
$$

where

$$
P(z):=\prod_{\substack{p \in \mathscr{D} \\ p<z}} p
$$

Estimates of $S(\mathscr{A}, \mathscr{P}, z)$ typically rely on the following standard regularity conditions on $\mathscr{A}$ and $\mathscr{P}$ (see [HR] for a detailed discussion). Let $R_{d}:=$ $\left|\mathscr{A}_{d}\right|-(\omega(d) / d) X$ and $\left|\mathscr{A}_{d}\right|:=\#\{a \in \mathscr{A}: d \mid a\}$ where $X$ is an approximation 
to $|\mathscr{A}|$ and $\omega(\cdot)$ is a nonnegative multiplicative arithmetic function such that $\omega(1)=1$ and $\omega(p)=0$ for all primes $p \notin \mathscr{P}$. Also assume $0 \leq \omega(p) / p \leq$ $1-1 / A_{1}$ for all $p \in \mathscr{P}$ and

$$
-L \leq \sum_{w \leq p<z} \frac{\omega(p)-\kappa}{p} \log p \leq A_{2} \quad(2 \leq w<z)
$$

where $A_{1}, A_{2}$, and $L$ are constants $>1$. The parameter $\kappa$ is known as the dimension of the sieve. Define $V(z):=\Pi_{p \mid P(z)}(1-\omega(p) / p)$, which can be considered intuitively as the probability that an element of $\mathscr{A}$ is not divisible by any prime $p<z$ with $p \in \mathscr{P}$.

Example A. Selberg's upper bound sieve [HR, Ra]. If $\kappa \geq 0, u=\log \xi^{2} / \log z>$ 0 , and $\nu(d)$ is the number of distinct prime divisors of $d$, then under the above hypothesis we have

$$
S(\mathscr{A}, \mathscr{P} ; z) \leq X V(z) \frac{1}{\sigma_{\kappa}(u)}\left\{1+O\left(\frac{(\log \log \xi)^{2 \kappa+1}}{\log \xi}\right)\right\}+\sum_{\substack{d<\xi^{2} \\ d \mid P(z)}} 3^{\nu(d)}\left|R_{d}\right|
$$

where $\sigma_{\kappa}(u):=j_{\kappa}(u / 2)$ is the Ankeny-Onishi-Selberg function. The $O$-constant depends only on $\kappa, A_{1}$, and $A_{2}$. Rawsthorne [Ra] showed that the left inequality in (8.1) can be omitted.

Example B. The sieve of Eratosthenes-Legendre [I1]. Similarly, if $0<\kappa<1 / 2$ then

$$
S(\mathscr{A}, \mathscr{P}, z)=X V(z)\left\{j_{-\kappa}(u)+O\left(\frac{(u+1) L}{1-2 \kappa}(\log z)^{2 \kappa-1}\right)\right\}
$$

where $u=\log x / \log z$ and $x:=\max _{a \in \mathscr{A}}|a|$. We must also assume $x \leq A_{3} X$ and $\left|R_{d}\right| \leq A_{4} \omega(d)$ for some constants $A_{3}, A_{4} \geq 1$. The $O$-constant depends only on $A_{1}, A_{2}, A_{3}$, and $A_{4}$.

Example C. Integers with large prime divisors. If

$$
\phi(x, y):=\#\{n \leq x: p \mid n \Rightarrow p \geq y\}
$$

then

$$
\phi(x, y) \sim x j_{-1}(u) \prod_{p<y}\left(1-\frac{1}{p}\right) \quad\left(x \rightarrow \infty, y=x^{1 / u}, u>1\right) .
$$

Note that $\phi(x, y)$ is a sifting function with $\mathscr{A}=\{n \leq x\}, \mathscr{P}=$ all primes, $z=y$, and $\kappa=1$. This result is originally due to Buchstab (see [Bu] or [dB1]). Since $\prod_{p<y}(1-1 / p) \sim e^{-\gamma} / \log y$ (Mertens' estimate), the result usually appears as $\phi(x, y) \sim x w(u) / \log y$ where $w(u)=e^{-\gamma} j_{-1}(u)$. This is an example of how "Mertens-type" information is sometimes incorporated in the definition of $p(u, a, b)$. Recently, Goldston and McCurley [GM2] have extended this result to $\phi(x, y, \mathscr{P}):=\#\{n \leq x: p \mid n, p \in \mathscr{P} \Rightarrow p>y\}$ where $\mathscr{P}$ is a set of primes 
that has relative density $\delta$ among the primes. They give a uniform result but we state only the asymptotic estimate

$$
\phi\left(x, x^{1 / u}, \mathscr{P}\right) \sim x j_{-\delta}(u) \prod_{\substack{p \leq y \\ p \in \mathscr{P}}}\left(1-\frac{1}{p}\right)
$$

as $x \rightarrow \infty$ and $u>0$ is fixed. Note that when $0<\delta<1 / 2$ this asymptotic estimate also follows from Example B.

Example D. Integers with small prime divisors. Let $u=\log x / \log y$ and let $\rho(u)=e^{\gamma} j_{1}^{\prime}(u)=e^{\gamma} p(u, 0,1)$ denote the well-known Dickman function. If

$$
\psi(x, y):=\#\{n \leq x: p \mid n \Rightarrow p \leq y\}
$$

then

$$
\psi(x, y)=x \rho(u)\left(1+O_{\varepsilon}\left(\frac{\log (u+1)}{\log y}\right)\right)
$$

holds uniformly in the range $x \geq 2$ and $\exp \left((\log \log x)^{5 / 3+\varepsilon}\right)<y \leq x$. This result is due to Hildebrand [Hi]. In [GM1], Goldston and McCurley generalized this result by considering

$$
\psi(x, y, \mathscr{P}):=\#\{n \leq x: p \mid n, p \in \mathscr{P} \Rightarrow p \leq y\}
$$

where $\mathscr{P}$ is a set of primes with relative density $\delta$ among the primes. If $\#\{p \leq x: p \in \mathscr{P}\}=\delta l i(x)+O\left(x /(\log x)^{B}\right)$ for constants $0<\delta<1$ and $B>2$, and $\rho_{\delta}(u)=e^{\delta \gamma} p(u, 0, \delta)$, then

$$
\psi(x, y, \mathscr{P})=x \rho_{\delta}(u)(1+O(1 / \log y))
$$

holds uniformly in the range $u \geq 1$ and $y \geq 1.5$. This is a nice example of the occurrence of $p(u, a, b)$ when $a+b \notin \mathbf{Z}$.

Example E. An incomplete sum of a multiplicative function [dKH]. If $0<\alpha<$ 2 and $u>0$ are fixed then

$$
\sum_{\substack{n \leq x \\ p \mid n \Rightarrow p \leq y}} \alpha^{\Omega(n)} \sim x(\log x)^{\alpha-1} e^{\alpha \gamma^{\prime}} u^{1-\alpha} j_{\alpha}^{\prime}(u) \prod_{p} \frac{\left(1-p^{-1}\right)^{\alpha}}{1-\alpha / p} \quad\left(x \rightarrow \infty, y=x^{1 / u}\right)
$$

where $\Omega(n)$ is the total number of prime divisors of $n$ counting multiplicity. For a general discussion of incomplete sums of multiplicative functions see [dBvL].

\section{FURTHER INTEGRAL TRANSFORMS}

We have seen that the adjoint relation (1.3) gives a link between the local behaviors of $p(u)$ and $q(u)$. Now we consider integral relationships between $p(u)$ and $q(u)$ that take into account their global behavior. This is done by considering $q(t+u)$ as the kernel of an integral transform in the variable $t$. What makes this interesting is that the mapping $f \mapsto F$ given by

$$
F(u)=\int_{0}^{\infty} f(t) q(t+u) d t
$$


maps a $p$-function into a constant multiple of a $q$-function when this integral converges.

Theorem 6. Let $u>0$.

(i) If $\alpha<1$ and $a+b<\alpha+\beta$ then

$$
\int_{0}^{\infty} p(t, \alpha, \beta) q(t+u, a, b) d t=\frac{\Gamma(\alpha+\beta-a-b)}{\Gamma(1-a-b)} q(u, a-\alpha+1, b-\beta) .
$$

(ii) If $m \geq 0$ and $\kappa>m$ then

$$
\int_{0}^{\infty} j_{\kappa}^{(m+1)}(t) q(t+u, a, b) d t=(-1)^{m} \frac{\Gamma(a+b)}{\Gamma(a+b-m)} q(u, a+\kappa-m, b-\kappa) .
$$

Proof. (i) For the integral in (i), $\alpha<1$ guarantees convergence at $t=0$ by (3.2) and (3.4), and $a+b<\alpha+\beta$ gives convergence at $t=\infty$ by (2.3) and Theorem 3(i). First assume $a+b<1$. Since $a+b<1$ we use (2.2) and the Laplace transform in Theorem 2(i) to get

$$
\begin{aligned}
\int_{0}^{\infty} & p(t, \alpha, \beta) q(t+u, a, b) d t \\
& =\frac{1}{\Gamma(1-a-b)} \int_{t=0}^{\infty} \int_{z=0}^{\infty} p(t, \alpha, \beta) z^{-a-b} e^{-(t+u) z+b \operatorname{Ein}(z)} d z d t \\
& =\frac{1}{\Gamma(1-a-b)} \int_{z=0}^{\infty} z^{-a-b} e^{-u z+b \operatorname{Ein}(z)} \int_{t=0}^{\infty} p(t, \alpha, \beta) e^{-t z} d t d z \\
& =\frac{1}{\Gamma(1-a-b)} \int_{0}^{\infty} z^{-(a-\alpha+1)-(b-\beta)} e^{-u z+(b-\beta) \operatorname{Ein}(z)} d z .
\end{aligned}
$$

Again (2.2) gives the result. The interchange of the order of integration is valid because of absolute convergence. For the case $a+b \geq 1$ note that as long as $\alpha<1$ and $a+b<\alpha+\beta$ this case follows by analytic continuation in $a$ and $b$, since both sides of the equation in (i) are analytic in $a$ and $b$.

(ii) Since $j_{\kappa}^{(m+1)}(t)=p(u, m+1-\kappa, \kappa)$, the case $a+b<m+1$ follows directly from (i) after using the reflection formula $\Gamma(z) \Gamma(1-z)=\pi / \sin (\pi z)$. Now the case $a+b \geq m+1$ follows by analytic continuation in $a$ and $b$, as in the proof of (i), because $j_{\kappa}^{(m+1)}(t)=O\left(e^{-t \log t}\right)$ implies that the integral in (ii) converges at $t=\infty$ for all $a$ and $b$. Actually, (ii) can be shown directly without using (i) and analytic continuation. Since the Laplace transform Theorem 2(ii) holds in all of $\mathbf{C}$, simply insert this into definition (2.1) and then show that reversing the order of integration is valid.

We now look at a special case of Theorem 6 for which $u$ can be arbitrary. This is the case when $p(t) \in\left\{j_{\kappa}^{(m+1)}(t)\right\}_{m=0}^{\infty}$ and $q(t) \in\left\{Q_{n}(t, \kappa)\right\}_{n=0}^{\infty}$ for some fixed $\kappa$. With the inner product $\int_{0}^{\infty} p(t) q(t) d t$ these two families of functions are almost orthogonal in the sense that this inner product is zero unless $m=n$, i.e., unless the two functions are adjoint. The "almost" is stated because the condition $\kappa>m$ cannot be relaxed. Although the following theorem can be deduced from Theorem 6(ii) using analytic continuation in $u$, we will give an interesting alternative proof using generating functions. Then a "discretized" version of some of these integrals will be presented. 
Theorem 7. Let $m, n \geq 0$ and $\kappa>m$; then

$$
\int_{0}^{\infty} Q_{n}(t, \kappa) j_{\kappa}^{(m+1)}(t) d t= \begin{cases}(-1)^{n} n ! & \text { if } n=m, \\ 0 & \text { otherwise. }\end{cases}
$$

Lemma 1. Let $g(t)=\sum_{n=0}^{\infty} g_{n} t^{n} / n$ ! be a formal power series. Define the infinitedimensional lower triangular matrix $\mathbf{M}(g)=\left[M_{n, m}(g)\right]_{n, m \geq 0}$ by the expansion

$$
e^{u t} g(t)=\sum_{n=0}^{\infty} \sum_{m=0}^{n} M_{n, m} u^{m} \frac{t^{n}}{n !} .
$$

Then for formal power series $f(t), g(t)$, and $h(t)$ we have

$$
f(t)=g(t) h(t) \Leftrightarrow \mathbf{M}(f)=\mathbf{M}(g) \mathbf{M}(h) .
$$

Proof. We first show the following:

$$
\begin{aligned}
M_{n, m}(g) & =\left.\frac{1}{m !} \frac{d^{n}}{d t^{n}} t^{m} g(t)\right|_{t=0}, \\
M_{n, m}(g) & =\left(\begin{array}{c}
n \\
m
\end{array}\right) g_{n-m}, \\
M_{n, m}(g(-t)) & =(-1)^{n+m} M_{n, m}(g(t)) .
\end{aligned}
$$

Differentiating (9.1) gives (9.2), which in turn gives (9.3) where $\left(\begin{array}{l}n \\ m\end{array}\right)=0$ for integers $m>n \geq 0$. Now Lemma 1 follows easily from (9.3) using the Cauchy product of two power series. We mention (9.4) because it will be used in the sequel and it follows from (9.1) with the substitutions $u \mapsto-u$ and $t \mapsto-t$.

Remark. This result is very reminiscent of the beautiful theorem on composition of power series: $f(t)=g(h(t)) \Leftrightarrow \mathbf{M}(f)=\mathbf{M}(h) \mathbf{M}(g)$. Here $f, g$, and $h$ are formal power series without a constant term and $\mathbf{M}$ is defined by (9.1) except with $e^{u g(t)}$ replacing $e^{u t} g(t)$. Also, it is interesting to note that Lemma 1 , along with (9.4), gives the binomial inversion formula when $g(t)=e^{t}$ and $h(t)=e^{-t}$.

Proof of Theorem 7. Let $g(t)=e^{\kappa \operatorname{Ein}(-t)}$ so that (2.6) and (9.1) imply $Q_{n}(u, \kappa)$ $=\sum_{\nu=0}^{n} M_{n, \nu}(g) u^{\nu}$. Consider the moments $\mu_{\nu, m}=\int_{0}^{\infty} t^{\nu} j_{\kappa}^{(m+1)}(t) d t$ for $\kappa>$ $m$ and recall that

$$
\int_{0}^{\infty} e^{s t} j_{\kappa}^{(m+1)}(t) d t=s^{m} e^{-\kappa \operatorname{Ein}(s)}=\frac{s^{m}}{g(-s)} \quad(\kappa>m) .
$$

Upon differentiating this equation $\nu$ times we get

$$
\frac{(-1)^{\nu}}{m !} \mu_{\nu, m}=\left.\frac{1}{m !} \frac{d^{\nu}}{d s^{\nu}} \frac{s^{m}}{g(-s)}\right|_{s=0}=M_{\nu, m}\left(\frac{1}{g(-s)}\right)=(-1)^{\nu+m} M_{\nu, m}\left(\frac{1}{g}\right) \text {. }
$$

Since $\mathbf{M}(g) \mathbf{M}(1 / g)$ equals the infinite-dimensional identity matrix we have for 
$\kappa>m$

$$
\begin{aligned}
\frac{(-1)^{m}}{m !} \int_{0}^{\infty} Q_{n}(t, \kappa) j_{\kappa}^{(m+1)}(t) d t & =\sum_{\nu=0}^{n} M_{n, \nu}(g) \frac{(-1)^{m}}{m !} \int_{0}^{\infty} t^{\nu} j_{\kappa}^{(m+1)}(t) d t \\
& =\sum_{\nu=0}^{n} M_{n, \nu}(g) M_{\nu, m}(1 / g) \\
& = \begin{cases}1 & \text { if } m=n, \\
0 & \text { otherwise. }\end{cases}
\end{aligned}
$$

Corollary 1. Let $m, n \geq 0, \kappa>m$, and $u$ be arbitrary. Then

$$
\int_{0}^{\infty} Q_{n}(t+u, \kappa) j_{\kappa}^{(m+1)}(t) d t= \begin{cases}(-1)^{m} m !\left(\begin{array}{c}
n \\
m
\end{array}\right) u^{n-m} & \text { if } m \leq n, \\
0 & \text { otherwise. }\end{cases}
$$

Proof. The result follows easily from Theorem 7 after noting that (2.7) gives

$$
Q_{n}(t+u, \kappa)=\sum_{\nu=0}^{n}\left(\begin{array}{l}
n \\
\nu
\end{array}\right) u^{n-\nu} Q_{\nu}(t, \kappa)
$$

Corollary 2. If $m \geq 0$ and $\kappa>m$ then

$$
\sum_{n=1}^{\infty} n j_{\kappa}^{(m+1)}(n) Q_{m}(n, \kappa)=(-1)^{m} \kappa m !
$$

Proof. In Corollary 1 put $u=1$ and $n=m$ and combine this with the adjoint relation (7.2) summed over $u=1,2,3, \ldots$.

Recall that $Q_{0}(u) \equiv 1$ and so Corollary 2 with $m=0$ gives us

$$
\sum_{n=1}^{\infty} n j_{\kappa}^{\prime}(n)=\kappa \quad(\kappa>0) .
$$

The case $\kappa=1$ where $j_{1}^{\prime}(u)=e^{-\gamma} \rho(u)$ was discovered numerically and proved by Knuth and Trabb Pardo [KTP]. They noted that this equality is an excellent check on the accuracy of computing $\rho(u)$. This remains true for the computation of $j_{\kappa}^{(m)}(u)$, at least for small $m$, since $Q_{m}$ is an easily computed polynomial.

A useful application of Theorem 6 is to represent a $q$-function as a generalized Stieltjes transform of a $p$-function.

Theorem 8. Let $u>0$.

(i) If $a<1$ and $a+b+\alpha>0$ then

$$
\int_{0}^{\infty} \frac{p(t, a, b)}{(t+u)^{\alpha+1}} d t=\frac{\Gamma(a+b+\alpha)}{\Gamma(\alpha+1)} q(u, 1-a-\alpha,-b) .
$$

(ii) If $m \geq 0$ and $\kappa>m$ then

$$
\int_{0}^{\infty} \frac{j_{\kappa}^{(m+1)}(t)}{(t+u)^{\alpha+1}} d t=(-1)^{m} \frac{\Gamma(-\alpha)}{\Gamma(-\alpha-m)} q(u, \kappa-m-\alpha,-\kappa) .
$$


Proof. (i) in Theorem 6(i) formally replace $(a, b, \alpha, \beta)$ with $(-\alpha, 0, a, b)$ and recall that $q(u,-\alpha, 0)=u^{-\alpha-1}$. Similarly, Theorem 6(ii) gives (ii).

Theorem 8 provides us with a proof, different from the one in [I2], of the fact that $q(u, a, b)>0$ when $b<0$ (see (2.5)). In Theorem 8(ii) let $m=0$, $\kappa>0$, and $\alpha$ be arbitrary. Since $j_{\kappa}^{\prime}(t)>0$ for $\kappa, t>0$, we get $q(u, a, b)>0$ for all $u>0$ whenever $b<0$. The other cases of (2.5), namely $a+b<$ $1, a+b=1$, and $b=0$, follow trivially from $(2.2), Q_{0}(u, b)=1$, and $q(u, a, 0)=u^{a-1}$, respectively.

\section{AN APPLICATION OF $q(u)$ IN NUMBER THEORY}

As was mentioned in the introduction and in $\S 8$, it has been known for many years that differential-difference equations of retarded type (such as (1.1)) can arise in the solution of certain types of problems in analytic number theory. Differential-difference equations of advanced type (such as (1.2)) have played only an auxiliary role (although an important one) in sieve methods. The following theorem is perhaps the first direct application to number theory of a function satisfying a differential-difference equation of advanced type.

Theorem 9. For $n \geq 1$ let $P_{1}(n)$ and $P_{2}(n)$ denote the largest and second largest prime divisor of $n$, respectively $\left(P_{1}(1)=1, P_{2}(n)=P_{1}\left(n / P_{1}(n)\right)\right)$. If $u \geq 1$ and $\alpha \in \mathbf{R}$ are fixed then

$$
\sum_{\substack{1<n \leq x \\ P_{2}(n) \leq P_{1}(n)^{1 / u}}}\left(\log P_{1}(n)\right)^{\alpha}=e^{\gamma} u^{\prime \gamma} q(u, 1-\alpha,-1) x(\log x)^{\alpha}+O\left(x(\log x)^{\alpha-1}\right) .
$$

Before giving the proof, we would like to make a few observations. First, note that (2.3) implies $e^{\gamma} u^{\alpha} q(u, 1-\alpha,-1) \sim e^{\gamma} / u$ as $u \rightarrow \infty$. In Theorem 8(ii) put $m=0$ and $\kappa=1$ and recall $\rho(u)=e^{\gamma} j_{1}^{\prime}(u)$ to get

$$
e^{\gamma} u^{\prime \alpha} q(u, 1-\alpha,-1)=u^{\alpha} \int_{0}^{\infty} \frac{\rho(t)}{(t+u)^{\alpha+1}} d t \quad(u>0)
$$

In a very interesting paper, Knuth and Trabb Pardo [KTP] discuss Golomb's constant $\lambda=0.6243299885 \ldots$, defined to be $\lim _{n \rightarrow \infty} l_{n} / n$ where $l_{n}$ is the average length of the longest cycle in a random $n$-permutation (see [GWG]). An interesting number theoretic interpretation of $\lambda$ is given by the fact that if $n$ is an $m$-digit integer then $\lambda m$ is the asymptotic average number of digits in 
$P_{1}(n)$. They also make reference to the following:

$(10.1)$

$\operatorname{Pr}\left\{P_{2}(n) \leq \sqrt{P_{1}(n)}\right\}=\lambda=\int_{0}^{\infty} \frac{\rho(t)}{t+2} d t \quad$ (heuristic proof in [KTP]),

$\sum_{n \leq x} \log P_{1}(n)=\lambda x \log x+O(x), \quad \lambda=\int_{0}^{\infty} \frac{\rho(t)}{(t+1)^{2}} d t \quad$ (de Bruijn [dB4]),

$$
\lambda=\int_{0}^{\infty} e^{-t-\mathrm{E}_{1}(t)} d t \quad \text { (Shepp and Lloyd [SL]). }
$$

Here, $\operatorname{Pr}\{S(n)\}$ is to be interpreted as the asymptotic density

$$
\lim _{n \rightarrow \infty} \#\{n \leq x: S(n) \text { is true }\} / x
$$

whenever $S(n)$ is a statement about the positive integers. All three of these results are encompassed in Theorem 9 . Recall that one of the auxiliary functions in sieve theory is denoted by $p_{\kappa}(u):=q(u, \kappa,-\kappa)$. If $\alpha=0$ and $u \geq 1$ then Theorem 9 implies

$$
\begin{aligned}
\operatorname{Pr}\left\{P_{2}(n) \leq P_{1}(n)^{1 / u}\right\} & =e^{\gamma} p_{1}(u)=\int_{0}^{\infty} \frac{\rho(t)}{t+u} d t \\
& =e^{\gamma} \int_{0}^{\infty} e^{-u t-\operatorname{Ein}(t)} d t
\end{aligned}
$$

where $p_{1}(u)=q(u, 1,-1)$ and satisfies $u p_{1}^{\prime}(u)=-p_{1}(u+1)$. Compare this with $\operatorname{Pr}\left\{P_{1}(n) \leq n^{1 / u}\right\}=\rho(u)$, which satisfies $u \rho^{\prime}(u)=-\rho(u-1)$. Equation (10.4) can be derived from the general result in [Bi]. For the case $\alpha=1$ note that (2.4) and (1.2) imply $u q(u, 0,-1)=p_{1}(u+1)$. In terms of the Dickman function this is

Thus Theorem 9 with $\alpha=1$ gives

$$
e^{\gamma} u q(u, 0,-1)=u \int_{0}^{\infty} \frac{\rho(t)}{(t+u)^{2}} d t=\int_{0}^{\infty} \frac{\rho(t)}{t+u+1} d t .
$$

$$
\sum_{\substack{n \leq x \\ P_{2}(n) \leq P_{1}(n)^{1 / u}}} \log P_{1}(n)=e^{\gamma} p_{1}(u+1) x \log x+O(x) \quad(u \geq 1) .
$$

Recall that $\operatorname{Ein}(t)=\gamma+\log t+E_{1}(t)$ for $t>0$, so that (2.2) gives us

$$
e^{\gamma} u q(u, 0,-1)=u \int_{0}^{\infty} e^{-u t-E_{1}(t)} d t \quad(u>0) .
$$

Thus, setting $u=2$ in (10.4) gives (10.1) and setting $u=1$ in (10.5) and (10.6) gives (10.2) and (10.3), respectively. Also, the three representations for $\lambda$ (along with a new one found in (10.4)) are all derived from $\lambda=e^{\gamma} p_{1}(2)$.

\section{Proof of Theorem 9}

Lemma 1 (de Bruijn [dB4]). There exist positive absolute constants $C_{1}$ and $C_{2}$ such that

$$
\psi(x, y)<C_{1} x e^{-C_{2} \log x / \log y} \quad(x>1, y \geq 2),
$$

where $\psi(x, y):=\#\left\{n \leq x: P_{1}(n) \leq y\right\}$. 
Lemma 2 (de Bruijn [dB4]). If $\varepsilon$ is a fixed positive number then

$$
\psi\left(x, x^{1 / v}\right)=x \rho(v)\left(1+O_{\varepsilon}(v \log (v+1) / \log x)\right)
$$

holds uniformly in the range $x \geq 3$ and $1 \leq v \leq(\log x)^{3 / 8-\varepsilon}$.

Let $\alpha_{0}=\max (\alpha, 0)$ and $y=\exp \left(\beta(\log x)^{1-\delta}\right)$ where $\beta=u(u+1)^{3 / 8-\varepsilon}$ and $0<\delta \leq 3 / 8-\varepsilon$ is fixed. Using Lemma 1 we have

$$
\begin{aligned}
\sum_{\substack{1<n \leq x \\
P_{2}(n)^{4} \leq P_{1}(n) \leq y}}\left(\log \left(P_{1}(n)\right)^{\alpha}\right. & \ll(\log y)^{\alpha_{0}} \psi(x, y) \\
& \ll \beta^{\alpha_{0}} x(\log x)^{(1-\delta) \alpha_{0}} \exp \left(\frac{-C_{2}}{\beta}(\log x)^{\delta}\right),
\end{aligned}
$$

which certainly falls within the error term of Theorem 9. Now

$$
\begin{aligned}
S & :=\sum_{\substack{1<n \leq x \\
P_{2}(n) \leq P_{1}(n)^{1 / u} \\
y<P_{1}(n)}}\left(\log P_{1}(n)\right)^{\alpha} \\
& =\sum_{y<p \leq x} \sum_{\substack{m \leq x / p \\
P_{1}(m) \leq p^{1 / u}}}(\log p)^{\alpha}=\sum_{y<p \leq x}(\log p)^{\alpha} \psi\left(\frac{x}{p}, p^{1 / u}\right) .
\end{aligned}
$$

Let $v(p)=u \log (x / p) / \log p$ and $z=x^{u /(u+1)}$. For $z<p \leq x$ we have $0 \leq v(p)<1, \rho(v(p))=1$, and $\psi\left(x / p, p^{1 / u}\right)=[x / p]$, so that

$$
\sum_{z<p \leq x}(\log p)^{\alpha} \psi\left(\frac{x}{p}, p^{1 / u}\right)=\sum_{z<p \leq x}(\log p)^{\alpha} \frac{x}{p} \rho(v(p))+O\left(\sum_{z<p \leq x}(\log p)^{\alpha}\right) .
$$

The error term on the right-hand side of $(11.1)$ is $\ll(\log x)^{\alpha} \pi(x) \ll x(\log x)^{\alpha-1}$. For $y<p \leq z$ we have

$$
1 \leq v(p)<u \log x / \log y=(u+1)^{\varepsilon-3 / 8}(\log x)^{\delta}
$$

and so

$$
(\log x / p)^{3 / 8-\varepsilon} \geq(u+1)^{\varepsilon-3 / 8}(\log x)^{3 / 8-\varepsilon}>v(p) .
$$

Thus we can use Lemma 2 in the range $y<p \leq x^{u /(u+1)}$. Combining this with (11.1) we get

$$
\begin{aligned}
S= & \sum_{y<p \leq x}(\log p)^{\alpha} \frac{x}{p} \rho(v(p)) \\
& +O\left(\sum_{y<p \leq z}(\log p)^{\alpha-1} \frac{x}{p} \rho(v(p)) \log (v(p)+1)\right)+O\left(x(\log x)^{\alpha-1}\right) .
\end{aligned}
$$


Let $S_{1}$ and $S_{2}$ denote the first and second sums, respectively, on the right-hand side of this equation. Using the prime number theorem in the form

$$
R(x):=\pi(x)-l i(x)=O\left(x e^{-c \sqrt{\log x}}\right)
$$

we have

$$
\begin{aligned}
\frac{S_{1}}{x(\log x)^{\alpha}} & =\int_{y}^{x}\left(\frac{\log s}{\log x}\right)^{\alpha} \rho(v(s)) \frac{d \pi(s)}{s} \\
& =-u^{\alpha} \int_{0}^{v(y)} \frac{\rho(t)}{(t+u)^{\alpha}} x^{-u /(t+u)} d \pi\left(x^{u /(t+u)}\right) \quad(t=v(s)) \\
& =u^{\alpha} \int_{0}^{v(y)} \frac{\rho(t)}{(t+u)^{\alpha+1}} d t-u^{\alpha} \int_{0}^{v(y)} x^{-u /(t+u)} d R\left(x^{u /(t+u)}\right) \\
& =u^{\alpha} \int_{0}^{\infty} \frac{\rho(t)}{(t+u)^{\alpha+1}} d t+O\left(\exp \left(-(\log x)^{\delta}\right)\right)+I
\end{aligned}
$$

The $O$-term follows from $\rho(t) \ll \exp (-t \log t)$ and $v(y)=(u+1)^{\varepsilon-3 / 8}(\log x)^{\delta}-$ $u$. We estimate $I$ by using (11.2) after an integration by parts. Let $f(t)$ denote the integrand in $I$ and note that $f(t) \ll x^{-u /(t+u)}$ and $f^{\prime}(t) \ll x^{-u /(t+u)} \log x$. Thus

$$
\begin{aligned}
I & \ll \frac{R(y)}{y}+\frac{R(x)}{x}+\int_{0}^{v(y)} R\left(x^{u /(t+u)}\right) x^{-u /(t+u)} \log x d t \\
& \ll(\log x)^{1+\delta} \exp \left(-c(\log x)^{(1-\delta) / 2}\right) .
\end{aligned}
$$

Essentially the same argument used to estimate $S_{1} / x(\log x)^{\alpha}$ will show that $S_{2} / x(\log x)^{\alpha-1}$ is bounded. This completes the proof of Theorem 9 .

\section{The ASYMPTOTIC EXPANSION OF $q(u)$}

In general, it is difficult to establish good inequalities for $q(u, a, b)$ and the techniques used are often ad hoc. Such inequalities can be very useful, especially in the study of the auxiliary functions $p_{\kappa}(u)$ and $q_{\kappa}(u)$ of sieve theory. We now present a representation for $p_{\kappa}(u)=q(u, \kappa,-\kappa)$ that can be used to obtain inequalities for $p_{\kappa}(u)$ and at the same time gives us another example of the many relationships between solutions to (1.1) and (1.2).

Theorem 10. If $u>0, \kappa>0, n \geq 0$, and $c \neq u$ then

$$
p_{\kappa}(u)=\sum_{m=0}^{n-1}(-1)^{m} \frac{Q_{m}(c,-\kappa)}{(u-c)^{m+1}}+\frac{(-1)^{n}}{(u-c)^{n}} \int_{0}^{\infty} j_{\kappa}^{\prime}(t) \frac{(t+c)^{n}}{t+u} d t .
$$

Proof. Let $H(t)=e^{-c t-\kappa \operatorname{Ein}(t)}$ and note that $H^{(n)}(t) \ll t^{-\kappa} e^{-c t}$ as $t \rightarrow \infty$ for all $n \geq 0$, giving us $\lim _{t \rightarrow \infty} e^{-(u-c) t} H^{(n)}(t)=0$. Now, using integration by 
parts repeatedly, we get

$$
\begin{aligned}
p_{\kappa}(u) & =\int_{0}^{\infty} e^{-u t-\kappa \operatorname{Ein}(t)} d t \\
& =\int_{0}^{\infty} e^{-(u-c) t} H(t) d t \\
& =\frac{1}{u-c} H(0)+\frac{1}{u-c} \int_{0}^{\infty} e^{-(u-c) t} H^{\prime}(t) d t \\
& =\sum_{m=0}^{n-1} \frac{H^{(m)}(0)}{(u-c)^{m+1}}+\frac{1}{(u-c)^{n}} \int_{0}^{\infty} e^{-(u-c) t} H^{(n)}(t) d t
\end{aligned}
$$

From (2.6) we see that $H^{(m)}(0)=(-1)^{m} Q_{m}(c,-\kappa)$. Using the Laplace transform in Theorem 2(ii) we have $H(s)=\int_{0}^{\infty} e^{-s(t+c)} j_{\kappa}^{\prime}(t) d t$ and so

$$
H^{(n)}(s)=(-1)^{n} \int_{0}^{\infty} e^{-s(t+c)}(t+c)^{n} j_{\kappa}^{\prime}(t) d t .
$$

Inserting this into the integral in (12.1) and reversing the order of integration (absolute convergence is obvious) immediately gives the result in Theorem 10.

Recall that $j_{\kappa}^{\prime}(t)>0$ for $\kappa, t>0$. Thus, simply by dropping the integral term, Theorem 10 gives a lower bound for $p_{\kappa}(u)$ whenever $n$ is even and an upper bound for $p_{\kappa}(u)$ when $n$ is odd and $0 \leq c<u$. Theorem 10 also gives us a method for choosing $c$ optimally in certain cases. For example, let $n=2$ and choose $c$ to be the zero of $Q_{1}(u,-\kappa)$, namely $c=-\kappa$, to get $p_{\kappa}(u) \geq 1 /(u+\kappa)$ with the error being $O\left((u+\kappa)^{-3}\right)$ as opposed to $O\left((u+\kappa)^{-2}\right)$. Theorem 10 does not seem to be easily generalized to $q(u, a, b)$ for arbitrary $(a, b)$, but we can generalize Iwaniec's [I2] asymptotic expansion of $q(u)$ using a remainder term similar to the one in Theorem 10. It is interesting to compare the expansion of $q(u)$ given below in Theorem 11 with the asymptotic expansion of $p(u, a, b)$ when $a+b \notin \mathbf{Z}$ given in Theorem 3 .

Theorem 11. If $c$ is a constant such that $u>c \geq 0$ and $n$ is a nonnegative integer such that $n>\max (a+b-2, b-1)$ then

$$
\begin{aligned}
q(u, a, b)= & \sum_{m=0}^{n} \frac{\Gamma(a+b)}{m ! \Gamma(a+b-m)} Q_{m}(c, b)(u-c)^{a+b-1-m} \\
& +(-1)^{n+1} \frac{\Gamma(n+2-a-b)}{\Gamma(1-a-b)} \int_{0}^{\infty} \frac{J_{n}(t-c,-b)}{(t+u-c)^{n+2-a-b}} d t
\end{aligned}
$$

where

$$
J_{n}(u, \kappa):=(-1)^{n+1}\left\{j_{\kappa}^{(-n)}(u)-\frac{(-1)^{n}}{n !} Q_{n}(-u,-\kappa)\right\} .
$$

Proof. We proceed as in [I2]. Hankel's contour integral for $1 / \Gamma(z)$ gives

$$
\frac{1}{2 \pi i} \int_{f} e^{s z} z^{\nu} d z=\frac{s^{-\nu-1}}{\Gamma(-\nu)} \quad(s>0) \text {. }
$$


Using this along with (2.1) and (2.6), we have for $u>c \geq 0$ and $n \geq 0$

$$
\begin{aligned}
q(u, a, b) & =\frac{\Gamma(a+b)}{2 \pi i} \int_{\mathscr{C}} z^{-a-b} e^{(u-c) z} e^{c z+b \operatorname{Ein}(-z)} d z \\
& =\frac{\Gamma(a+b)}{2 \pi i} \int_{\mathscr{C}} z^{-a-b} e^{(u-c) z} \sum_{m=0}^{n} Q_{m}(c, b) \frac{z^{m}}{m !} d z+R \\
& =\Gamma(a+b) \sum_{m=0}^{n} \frac{Q_{m}(c, b)}{m ! \Gamma(a+b-m)}(u-c)^{a+b-1-m}+R
\end{aligned}
$$

where

$$
R=\frac{\Gamma(a+b)}{2 \pi i} \int_{\mathscr{C}} z^{-a-b} e^{(u-c) z} R_{n}(z) d z
$$

and

$$
R_{n}(z)=e^{c z+b \operatorname{Ein}(-z)}-\sum_{m=0}^{n} Q_{m}(c, b) \frac{z^{m}}{m !} .
$$

Since $R_{n}(z) / z^{n+1}$ is entire and $n>a+b-2$, the integral $R$ converges at $z=0$. Thus, we deform the contour $\mathscr{C}$ into two negative half-lines with opposite orientations so that

$$
z^{-a-b}= \begin{cases}e^{-(a+b)(\log |z|-\pi i)} & \text { on the lower half-line } \\ e^{-(a+b)(\log |z|+\pi i)} & \text { on the upper half-line }\end{cases}
$$

Now

$$
\begin{aligned}
R & =\frac{\Gamma(a+b)}{2 \pi i} \int_{-\infty}^{0}|z|^{-a-b} e^{(u-c) z} R_{n}(z)\left\{e^{\pi(a+b) i}-e^{-\pi(a+b) i}\right\} d z \\
& =\frac{\Gamma(a+b) \sin (\pi(a+b))}{\pi} \int_{0}^{\infty} z^{-a-b} e^{-(u-c) z} R_{n}(-z) d z .
\end{aligned}
$$

Multiply the Laplace transform in Theorem 2(i) (with $(a, b)=(b-n,-b)$ ) by $e^{-c s}$ to get

$$
\int_{0}^{\infty} e^{-s t} j_{-b}^{(-n)}(t-c) d t=s^{-n-1} e^{-c s+b \operatorname{Ein}(s)}
$$

for all $\mathfrak{R} s>0, b<n+1$, and $c \geq 0$. Using (2.7) we have

$$
\int_{0}^{\infty} e^{-s t} \frac{(-1)^{n}}{n !} Q_{n}(c-t, b) d t=s^{-n-1} \sum_{m=0}^{n} \frac{(-1)^{m}}{m !} Q_{m}(c, b) s^{m} \quad(\Re s>0) .
$$

Subtracting (12.4) from (12.3) and noting the definitions of $J_{n}(u, \kappa)$ and $R_{n}(z)$ (see (12.2)), we get

$$
\int_{0}^{\infty} e^{-s t} J_{n}(t-c,-b) d t=(-1)^{n+1} s^{-n-1} R_{n}(-s) \quad(s \in \mathbf{C}, c \geq 0, b<n+1) .
$$

Thus

$$
R=\frac{(-1)^{n+1}}{\Gamma(1-a-b)} \int_{z=0}^{\infty} z^{n+1-a-b} e^{-(u-c) z} \int_{t=0}^{\infty} e^{-z t} J_{n}(t-c,-b) d t d z
$$


and reversing the order of integration immediately finishes the proof of Theorem 11.

Acknowledgments. I wish to thank Harold Diamond for his invaluable assistance throughout this research. I also want to thank Adolf Hildebrand for several very helpful conversations concerning Theorem 9.

\section{REFERENCES}

[AS] M. Abramowitz and I. A. Stegun, Handbook of mathematical functions, Wiley, New York, 1972.

[A] K. Alladi, The distribution of $\nu(n)$ in the sieve of Eratosthense, Quart. J. Math. Oxford Ser. (2) 33 (1982), 129-148.

[AO] N. C. Ankeny and H. Onishi, The general sieve, Acta Arith. 10 (1964), 31-62.

[Bi] P. Billingsley, On the distribution of large prime divisors, Period. Math. Hungar. 2 (1972), 283-289.

[BH] N. Bleistein and R. A. Handelsman, Asymptotic expansions of integrals, Dover, New York, 1975.

[dB1] N. G. de Bruijn, On the number of uncancelled elements in the sieve of Eratosthenes, Nederl. Akad. Wetensch. Proc. 53 (1950), 803-812.

[dB2] __ On some Volterra integral equations of which all solutions are convergent, Nederl. Akad. Wetensch. Proc. 53 (1950), 813-821.

[dB3] - The asymptotic behaviour of a function occurring in the theory of primes, J. Indian Math. Soc. 15 (1951), 25-32.

[dB4] On the number of positive integers $\leq x$ and free of prime factors $>y$, Nederl. Akad. Wetensch. Proc. Ser. A 54 (=Indag. Math. 13) (1951), 50-60.

[dBvL] N. G. de Bruijn and J. H. van Lint, Incomplete sums of multiplicative functions, I, II, Nederl. Akad. Wetensch. Proc. Ser. A 67 (=Indag. Math. 26) (1964), 339-347; 348-359.

[Bu] A. A. Buchstab, Asymptotic estimates of a general number-theoretic function, (Russian, German summary), Mat. Sb. 44 (1937), 1237-1246.

[Ca] E. R. Canfield, The asymptotic behavior of the Dickman-de Bruijn function, Congr. Numer. 35 (1982), 139-148.

[Ch] J. M. F. Chamayou, A probabilistic approach to a differential-difference equation arising in analytic number theory, Math. Comp. 27 (1973), 197-203.

[DH] H. Diamond and H. Halberstam, The combinatorial sieve, Proc. Ootacamund, India, Jan. 1984, Lecture Notes in Math., vol. 1122, Springer-Verlag, Berlin, 1985, pp. 63-73.

[DHR1] H. Diamond, H. Halberstam, and H.-E. Richert, Combinatorial sieves of dimension exceeding one, J. Number Theory 28 (1988), 306-346.

[DHR2] _ A boundary value problem for a pair of differential delay equations related to sieve theory, I (to appear).

[Do1] G. Doetsch, Guide to the applications of the Laplace and $\mathscr{B}$-transforms, Van Nostrand Reinhold, New York, 1971.

[Do2] _ Introduction to the theory and application of the Laplace transformation, translation by W. Nader, Springer-Verlag, New York, 1974.

[GM1] D. A. Goldston and K. S. McCurley, Sieving the positive integers by large primes, J. Number Theory 28 (1988), 94-115.

[GM2] $\_$Sieving the positive integers by small primes, Trans. Amer. Math. Soc. 307 (1988), $51-62$.

[GWG] S. W. Golomb, L. R. Welch, and R. M. Goldstein, Cycles from nonlinear shift registers, Prog. Report No. 20-389, Jet Propulsion Laboratory, California Institute of Technology, Pasadena, 1959. 
[G] F. Grupp, On difference-differential equations in the theory of sieves, J. Number Theory 24 (1986), 154-173.

[GR] F. Grupp and H.-E. Richert, Notes on functions connected with the sieve, Analysis 8 (1988), 1-23.

[HR] H. Halberstam and H.-E. Richert, Sieve methods, Academic Press, 1974.

[He] D. Hensley, The convolution powers of the Dickman function, J. London Math. Soc. (2) 33 (1986), 395-406.

[Hi] A. Hildebrand, On the number of positive integers $\leq x$ and free of prime factors $>y, \mathrm{~J}$. Number Theory 22 (1986), 289-307.

[I1] H. Iwaniec, The sieve of Eratosthenes-Legendre, Ann. Scuola Norm. Sup. Pisa Cl. Sci. (4) 2 (1977), 257-268.

[12] _ Rosser's sieve, Acta Arith. 36 (1980), 171-202.

[ILR] H. Iwaniec, J. van de Lune, and H. J. J. te Riele, The limits of Buchstab's iteration sieve, Nederl. Akad. Wetensch. Indag. Math. Proc. A 83 (4) (1980), 409-417.

[KTP] D. E. Knuth and L. Trabb Pardo, Theoret. Comput. Sci. 3 (1976), 321-348.

[dKH] J. M. de Koninck and D. Hensley, Sums taken over $n \leq x$ with prime factors $\leq y$ of $z^{\Omega(n)}$, and their derivatives with respect to $z$, J. Indian Math. Soc. (N.S.) 42 (1978-79), 353-365.

[LF] B. V. Levin and A. S. Fainleib, Applications of some integral equations to problems of number theory, Russian Math. Surveys 22 (1967), 119-204.

[M] H. Maier, Primes in short intervals, Michigan Math. J. 32 (1985), 221-225.

[O] F. W. J. Olver, Asymptotics and special functions, Academic Press, New York, 1974.

[vODN] M. P. van Ouwerkerk-Dijkers and J. Nuis, On the asymptotic behaviour of the solution of a differential-difference equation arising in number theory, Math. Centrum Amsterdam Afd. Toegepaste Wisk. Rep. TN 50 (1968), 9 pp.

[Ra] D. Rawsthorne, Selberg's sieve estimate with a one sided hypothesis, Acta Arith. 41 (1982), 281-289.

[SL] L. Shepp and S. P. Lloyd, Ordered cycle lengths in a random permutation, Trans. Amer. Math. Soc. 121 (1966), 340-357.

[U] S. Uchiyama, On a differential-difference equation, J. Fac. Sci. Hokkaido Univ. Ser. I 19 (1986), 59-65.

[W] F. S. Wheeler, On two differential-difference equations arising in analytic number theory, Ph.D. thesis, University of Illinois, 1988.

Supercomputing Research Center, 17100 Science Drive, Bowie, Maryland 20715-4300 